Ann. Zootech., I96I, 10 (4), 279-3II.

\title{
LES FACTEURS DE VARIATION DU RAPPORT CALORIES/MATIÈRES AZOTÉES TOTALES DANS LES RÉGIMES DU POUSSIN
}

\author{
J. GUILLAUME \\ Station de Recherches avicoles, \\ Centre national de Recherches zootechniques, Jouy-en-Josas (Seine-et-Oise).

\section{PLAN}

\section{INTRODUCTION}

\section{I. - IMPORTANCE DU RAPPORT CALORIES/MatTÈ̀ES AZOTÉES TOTALES}

Io Influence du rapport Cal/M. A. T. sur le développement pondéral de l'animal.

$2^{\circ}$ Influence du rapport Cal/M. A. T. sur l'efficacité des alimeits.

a) efficacité azotée ;

b) efficacité calorique ;

c) compensation de l'efficacité azotée et caloricue l'une par l'autre ;

d) signification de l'indice de consommation.

$3^{\circ}$ Influence du rapport Cal/M. A. T. sur la composition corporelle.

II. - LES VARIATIONS CAL/M. A. T. OPTIMAL

A) Facteurs liés à l'animal.
$\mathrm{I}^{\mathrm{O}}$ l'âge ;
$2^{0}$ le sexe;
$3^{0}$ l'origine génétique.

B) Facteurs liés au régime.

Aspect quantitatif :

$\mathbf{I}^{\mathrm{o}}$ Influence de la valeur absolue des termes du rapport sur sa valeur optimale.

a) cas de la croissance;

b) cas de l'efficacité alimentaire ;

c) cas de la composition corporelle.

$2^{\mathrm{O}}$ Rôle direct du taux énergétique.

a) action sur la croissance ;

b) interprétation ;

c) conclusion.

Aspect qualitatif :

$\mathbf{I}^{0}$ rôle de la nature des matières azotées;

$2^{0}$ rôle de la nature des matières énergétiques.

a) position du problème;

b) incertitude des mesures de l'énergie productive des matières grasses.

œ) Facteurs intrinsèques : Digestibilité des graisses sensu stricto.

Influence du taux de matière grasse.

Influence de la nature des matières grasses. 
Influence du point de fusion.

Influence du degré d'estérification.

ß) Facteurs extrinsèques.

c) rôle des constituants non énergéticues des matières grasses;

d) différences de rôle métabolique entre glucides et lipides.

CONCLusion

$3^{\circ}$ Discussion.

\section{INTRODUCTION}

La nécessité de fournir les différents éléments du régime dans un rapport défini a trouvé une application du plus haut intérêt depuis une dizaine d'années dans la relation qui doit exister entre les principes énergétiques et azotés.

Bien que cette notion soit assez ancienne puisque MOELl,gaARo (cité par HoH, s I958) et LEROY définissent les besoins azotés des différentes espèces par unité énergétique (unité fourragère), elle a été remise à l'honneur chez les volailles à la suite des travaux de Sco'T'T et al. (I947) qui soulignent l'intérêt de l'enrichissement des régimes en énergie, et de HiLl et DANsky (I950) qui étudient l'influence de la quantité de calories du régime sur sa consommation. Ces auteurs retrouvent l'importance de l'équilibre énergie/matières azotées pour l'amélioration de 1'efficacité des aliments.

Depuis cette époque, le nombre de publications dans ce domaine est considérable. L,a plus grande partie d'entre elles concerne des expériences à caractère pratique dont le but est de déterminer les modalités d'emploi des matières grasses dans l'alimentation des volailles. Bien peu, par contre, ont été poursuivies dans un esprit de recherches fondamentales.

Dans la plupart des cas, l'obligation de maintenir un rapport calories/matières azotées convenable est mise en évidence, mais de nombreuses imprécisions demeurent qui rendent les applications zootechniques souvent difficiles. Il y a lieu de considérer en effet que la variation de ce rapport peut être obtenue de plusieurs façons qui n'ont pas la même signification physiologique. Il nous est donc apparu opportun de faire le point de la question en tentant de dégager les causes de la variabilité des résultats par une analyse critique des données expérimentales publiées à ce jour.

On a proposé plusieurs modes d'expression de la relation entre l'énergie et les matières azotées. La plus répandue chez les volailles consiste à rapporter la valeur énergétique des régimes exprimée en calories productives (FraPs, I946) au pourcentage de matières azotées totales. Ce dernier terme est égal à l'azote analysé selon la méthode de KJELDAHI, multiplié par le coefficient 6,25. Par souci de commodité, nous utiliserons l'abréviation Cal/M. A. T. pour désigner ce rapport dans lequel les calories sont évaluées par kilogramme d'aliment. Dans ces conditions, la valeur moyenne de ce rapport oscille entre 70 et I 20.

\section{I. - IMPORTANCE DU RAPPORT CALORIES/MATIỲRES AZOTÉES TOTALES}

De nombreuses observations ont démontré l'influence du rapport Cal/M. A. T. sur le développement pondéral de l'animal, sur sa composition corporelle et sur l'efficacité alimentaire. 
I. - Infuence du rapport Cal/M. A.T. sur le développement pondéral de l'animal

Donaldson et al. (I956), étudient la réponse de croissance de poussins recevant des régimes dont le rapport Cal/M. A. T. passe de 77 à I56. Ils n'observent aucune variation du poids de l'animal tant que le rapport est inférieur à II5 (Courbes de DONALDSON et al. graphique I). Au-delà de cette limite, la croissance est toujours

- Calorie/g de gair.

* Croissance.

A Croissance (taux énercétiques fort et moyen).

- Protéines/g de gain

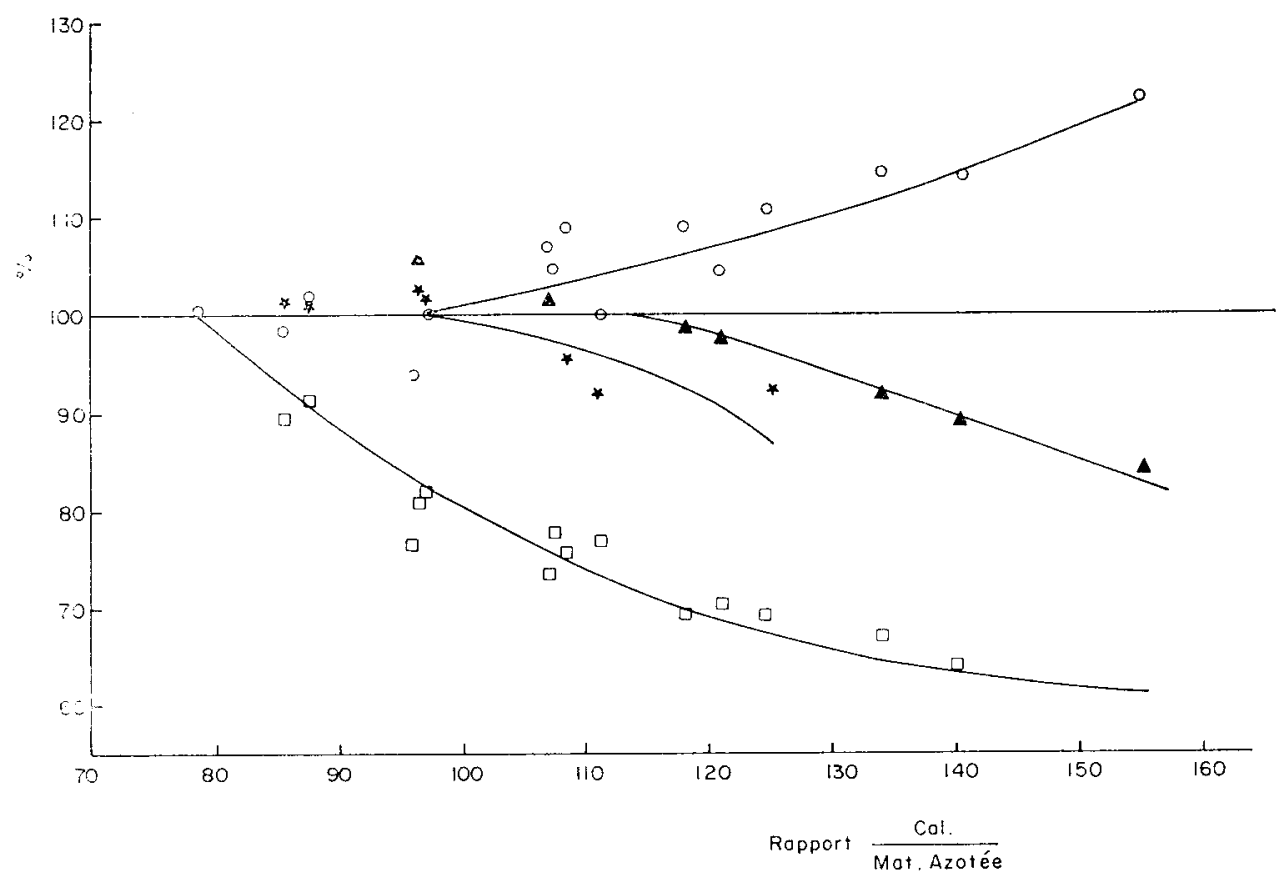

Graphique I. - Variations relatives en pourcentage de la croissance et de l'ingestion d'éléments nutritifs en fonction du rapport CallMatières azotées totales.

(Donaldson et al. I956, Poultry Science 35, page i 102, fig. I).

ralentie. Ce résultat est cependant incomplet : Hri et DANsky (r95o) avaient déjà montré que l'on peut obtenir une dépression de la croissance aussi bien quand on augmente le taux azoté au-delà d'une certaine valeur que l'orsqu'on le diminue au-dessous d'une autre valeur sans diminuer le taux calorique parallèlement; ce qui signifie que la dépression de croissance apparaît de part et d'autre de deux valeurs données du rapport Cal/M. A. T. Toutefois, à l'intérieur de ces limites, le rapport Cal/M. A. T. peut varier dans une large mesure sans avoir de répercussion significative sur le taux de croissance. Cette conclusion est renforcée par de très nombreux résultats identiques d'auteurs qui ont travaillé avec des valeurs plus étalées. On peut traduire ces faits par des courbes donnant le poids de l'animal à un âge donné en fonction du 
rapport Cal/M. A. T. (graphique 2). Ces courbes présentent toutes une forme en cloche dont le maximum n'est pas toujours bien défini. C'est en ce sens que VONDELL

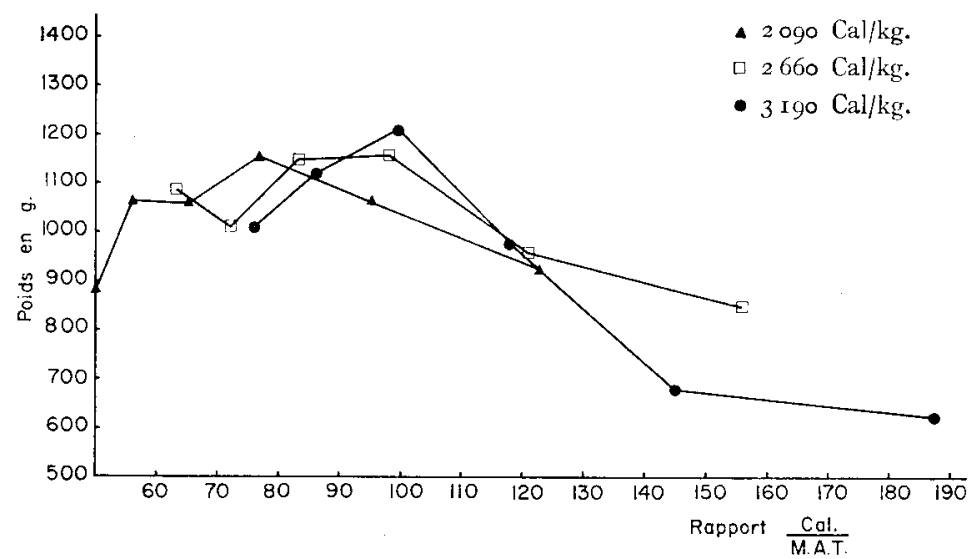

GRAPHIQUe 2. - Variations du poids vit moyen de lots de poulets de neuf semaines ayant rę̧u des régimes ì rapports CallM.A.T. différents (régimes isoénergétiques).

Établi d'après les données de :

LEONG et al. 1959, Poultry Science 38, 1267-1284. Exp. 3.

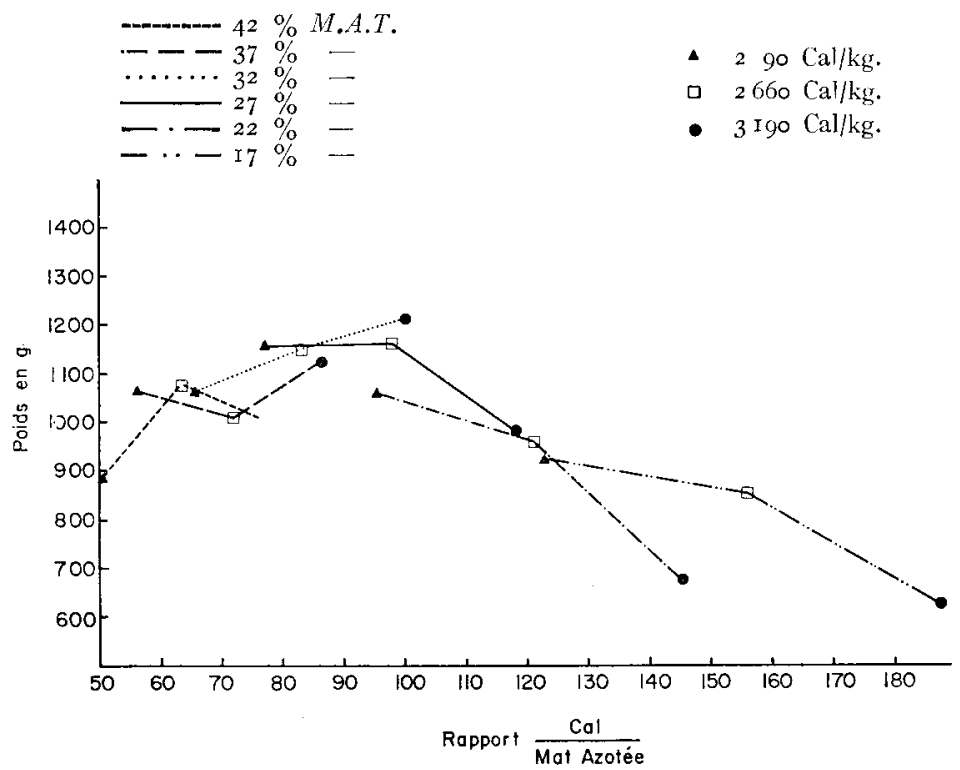

GRAPHIQUe 2 bis. -- Variations du poids vif moyen de lots de poulets de nent semaines ayant rę̧u des régime à rapports CallM.A.T. différents (régimes isopretéiques).

Etabli d'après les données de:

LeONG et al. 1959, Poultry Science, 38, 1267-1284, Exp. 3.

et Ringrose (I958) préfèrent parler d'une "zone " où la variation du rapport n'a pas d'influence significative sur la croissance. Pour ces auteurs, cette "zone "se situe entre les valeurs 99 et II7. Malgré ces imprécisions, la plupart des chercheurs définissent cependant une valeur optimale. Il convient toutefois d'ajouter, comme le 
fait HoHıs (I958) que l'on ne doit pas s'attendre à une précision supérieure à plus ou moins ro pour cent.

Ces données traduisent l'inaptitude du poussin à utiliser convenablement des régimes déséquilibrés aussi bien par excès que par déficience d'énergie ou de matières azotées. Notons en passant, qu'on ne trouve pas un seul maximum, mais bien autant de maximums que les auteurs utilisent de séries de régimes. Nous reviendrons sur ce point.

\section{2. - Infuence du rapport Cal/M. A.T. sur l'efficacité des alimenis}

On a essayé d'employer des aliments enrichis en énergie en vue d'améliorer la croissance et les résultats n'ont pas toujours été positifs. Par contre, on s'est vite aperçu que ces régimes pouvaient améliorer 1'indice de consommation (Yacowrtz, I953) et c'est sans doute l'intérêt pratique de cette constatation qui a encouragé les chercheurs dans cette voie.

L'augmentation de l'efficacité de la ration est le fait d'une élévation du taux de calories du régime car on obtient alors la même croissance avec une quantité inférieure d'aliment. On remarque inversement que la consommation d'aliment par unité de gain augmente lorsque l'on enrichit le régime en cellulose.

Toutefois, le poids d'aliments consommé par unité de gain corporel n'est pas un critère suffisant de l'utilisation des principes énergétiques et azotés. Il ne donne en effet qu'une valeur de l'utilisation globale de la totalité de la ration. Il serait plus intéressant de considérer le gain de poids par gramme de matières azotées totales ou par calorie consommée, c'est-à-dire l'efficacité azotée et l'efficacité calorique.

\section{a) Efficacité azotée.}

BIEly et March (I956) notent les premiers que l'élévation du taux énergétique du régime par adjonction de matières grasses se traduit par une économie des autres éléments nutritifs et en premier lieu des matières azotées totales. DoNALDSON et al. (I956) ont porté sur un graphique (graphique I) le nombre de grammes de matières azotées totales nécessaires à l'obtention d'un gramme de gain (inverse de l'efficacité protéique) en fonction du rapport Cal/M. A. T. La courbe qu'ils obtiennent a l'allure d'une branche d'hyperbole; plus le rapport s'élève, meilleure est l'efficacité azotée.

On sait que l'efficacité azotée dépend de plusieurs facteurs. HiLL et DANSK Y, (I 954) Mraz et al. (I958) puis LEONG et al. (I959) indiquent qu'elle diminue lorsque le taux protéique augmente. En particulier, ces derniers auteurs ne font que transposer au cas du poulet les résultats obtenus par BARNEs et BosshardT (I946), chez la souris. Ils fournissent par contre des indications supplémentaires puisque leurs conclusions demeurent valables lorsque l'on fait varier également le taux énergétique du régime.

On peut cependant exprimer leurs résultats de façon différente. Nous avons repris les chiffres des auteurs précédents et nous avons étudié le rapport M. A. 'T. consommées en fonction du rapport Cal/M. A. T. Les courbes obtenues sont comparables à des branches d'hyperboles (graphique 3) comme celles de DONALDSON et al. (I956). 
Si nous portons maintenant la relation inverse : $\frac{\text { gain corporel }}{\text { M.A.T consommées }}$ (coefficient d'efficacité protidique) en fonction du rapport Cal/M. A. T., nous obtenons une droite. Pour les valeurs du rapport inférieures à I20, il y a proportionnalité entre l'efficacité

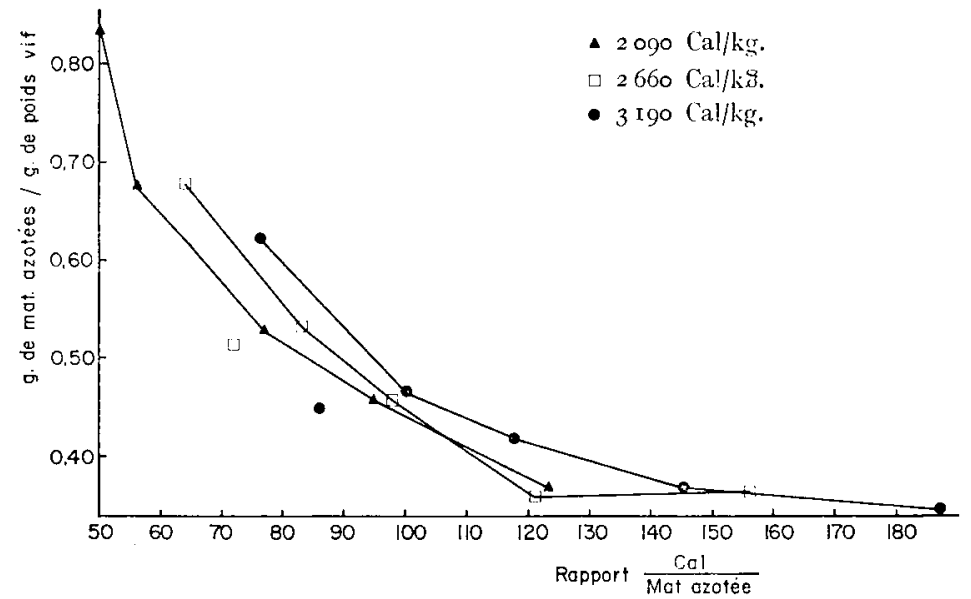

GRAPHIQUE 3. - Variations du rapport moyen: g de matières asotées totales ingérées/g de poids vif de lots de poulets ayanl resu des régimes à rapporls CalM.M.T. v'ariables.

Établi d'après les données de:

LEONg et al. 1959, Poultry Science, 38, 1267-128. lixp. г3.

azotée et le rapport calories/M. A. T. (graphique 4). Au-delà de la valeur I2o l'utilisation des matières azotées ne peut plus être améliorée.

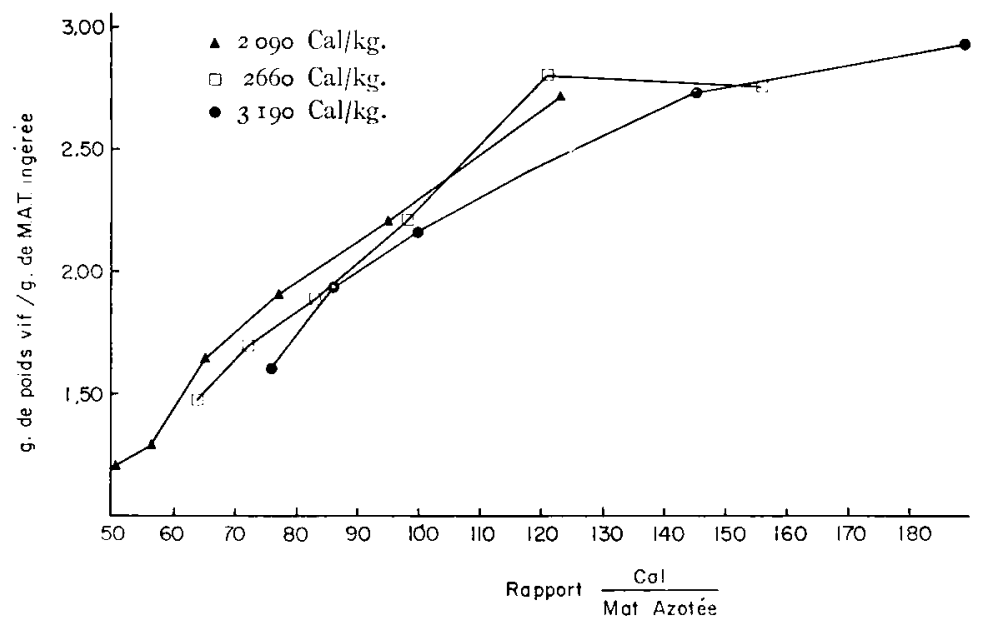

Graphique 4. - Variations du rappont moyen g de M.1.T. ingérées/kg de poids arif (efficacité protéique) de lots de poulets ayant recu des régimes a rapport CallM.A.T. variables.

Établi d'après les données de:

LEONG et al. I959, Poultry Science, 38, I267-т284. Exp. I3.

L'efficacité azotée maximale est donc observée pour des rapports Ca1/M. A. T. voisins de 220 . Ceci nous montre que pour des régimes où le rapport est inférieur à Ioo, les protéines ne sont pas en mesure de manifester leur pleine efficacité, l'animal ne disposant pas d'assez d'énergie pour cela. 
b) Efficacité calorique.

En plus de l'efficacité protéique, les auteurs précédemment cités ont étudié dans les mêmes conditions les variations de l'efficacité calorique.

Donal,DSON et al. (I956) observent que la quantité de calories ingérées par gramme de gain de poids augmente avec le rapport Cal/M. A. T. (graphique I). Il y a donc une variation en sens inverse des efficacités azotées et caloriques. Autrement dit, c'est en dépensant davantage de calories que le poulet utilise mieux les matières azotées du régime. Ces résultats doivent cependant être nuancés.

Bien que LEONG et al. (r959) n'aient pas publié leurs résultats sous la même forme que DONAL,DSON et al. (I956) nous avons pu, d'après leurs chiffres, tracer les

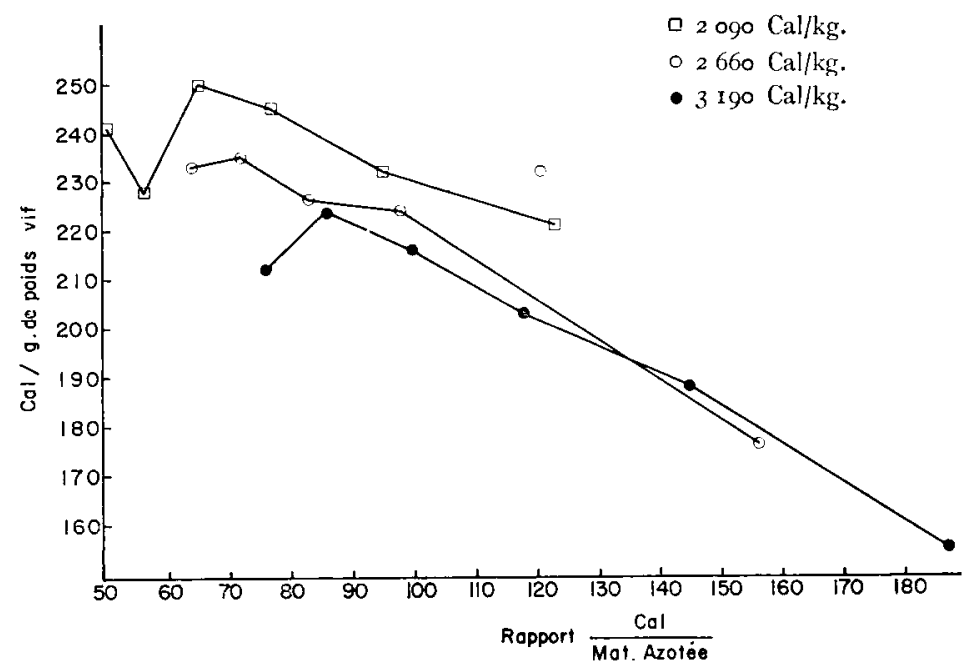

Graphique 5. -- Variations du rapport moyen Cal. productives ingérées/g de poids vif (efficacitécalorique de lots de poulets ayant reçu des régimes à rapports CallMAT variables.

Établi d'après les données de:

LEONG et al. 1959. Poultry Science, 38, I 267-1 284. Lxp. I3.

courbes de variation de l'efficacité calorique en fonction du rapport Cal/M. A. T. et nous les avons collationnés sur un graphique (graphique 5). Ces résultats ne sont pas entièrement superposables. Pour les faibles valeurs dı rapport Cal/M. A. 'T., DONALDSON et al. (I956), trouvent que l'efficacité calorique demeure constante, alors que pour LEONG et al. elle augmente, passe par un maximum avant de diminuer.

Cette contradiction apparente s'explique, là encore, par le fait que les deux études n'ont pas été poursuivies dans la même gamme de rapport Cal/M. A. 'T. C'est sans doute, parce que Donaldson et al. ne sont pas descendus en dessous des valeurs correspondant à l'optimum, que les conclusions diffèrent.

c) Compensation de l'efficacité azotée et calorique l'une par l'autre.

En résumé, les auteurs aboutissent aux conclusions suivantes :

I $^{\circ}$ L,e poulet utilise mieux les matières azotées totales lorsqu'il reçoit un tatıx calorique plus élevé dans son régime. Mais en contre partie il gaspille cette énergie excédentaire. 
$2^{\circ}$ Ce résultat n'est valable que dans la zone du rapport Cal/M. A. T. optimale.

En fonction des variations de ce rapport à un âge donné du poussin, nous avons schématisé sur un même graphique (figure 6) les variations simultanées de l'efficacité azotée, de l'efficacité calorique et du poids vif. On peut décomposer ce graphique en trois zones. Pour la zone centrale, les efficacités caloriques et azotées varient en sens inverse. L'élévation de l'une compense la réduction de l'autre. Par contre

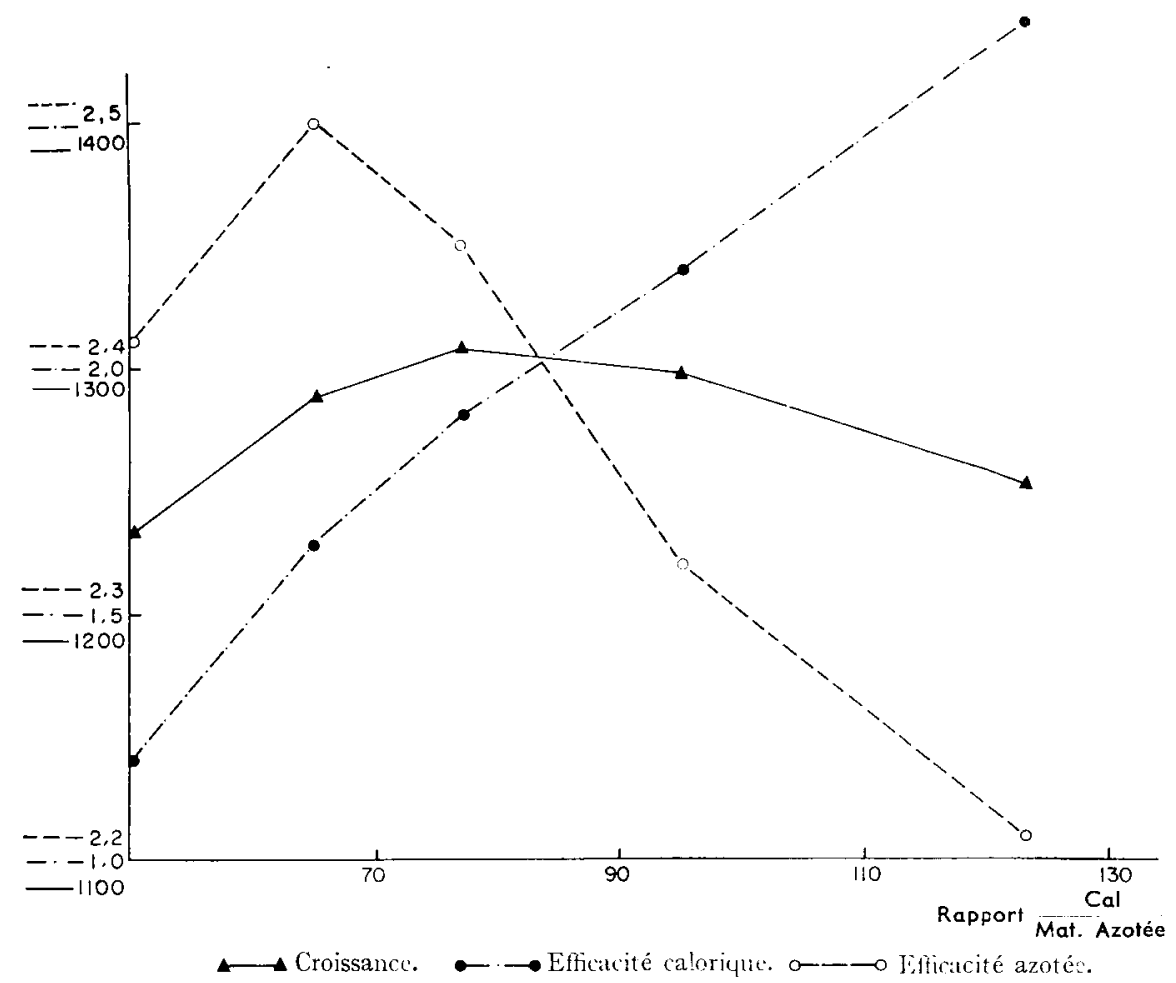

Graphique 6. -- Variations simultanées de la croissance, le l'efficacité azotée et de l'efficacité énergétique pour les valeurs du rapport CallM.A.T. oplimales.

Établi d'après les données de:

LEONG et al. 1959, Poullry Science, 38, 1267-1284. 1.xp. I3.

dans les zones marginales, pendant que l'une varie, l'autre demeure sensiblement constante. Il en résulte que, dans ces deux zones, l'un des constituants du régime est mal utilisé.

d) Signification de l'indice de consommation.

Comme le fait remarquer Hohrs (I958) on peut obtenir un même rapport Cal/M. A. T. de plusieurs manières qui n'ont pas cependant la même signification physiologique.

Isorsque $\mathrm{HoHI}_{\mathrm{s}}$ étudie la variation de l'indice de consommation en fonction du rapport Cal/M. A. T., il obtient deux courbes différentes, (graphique 7 et 7 bis), 


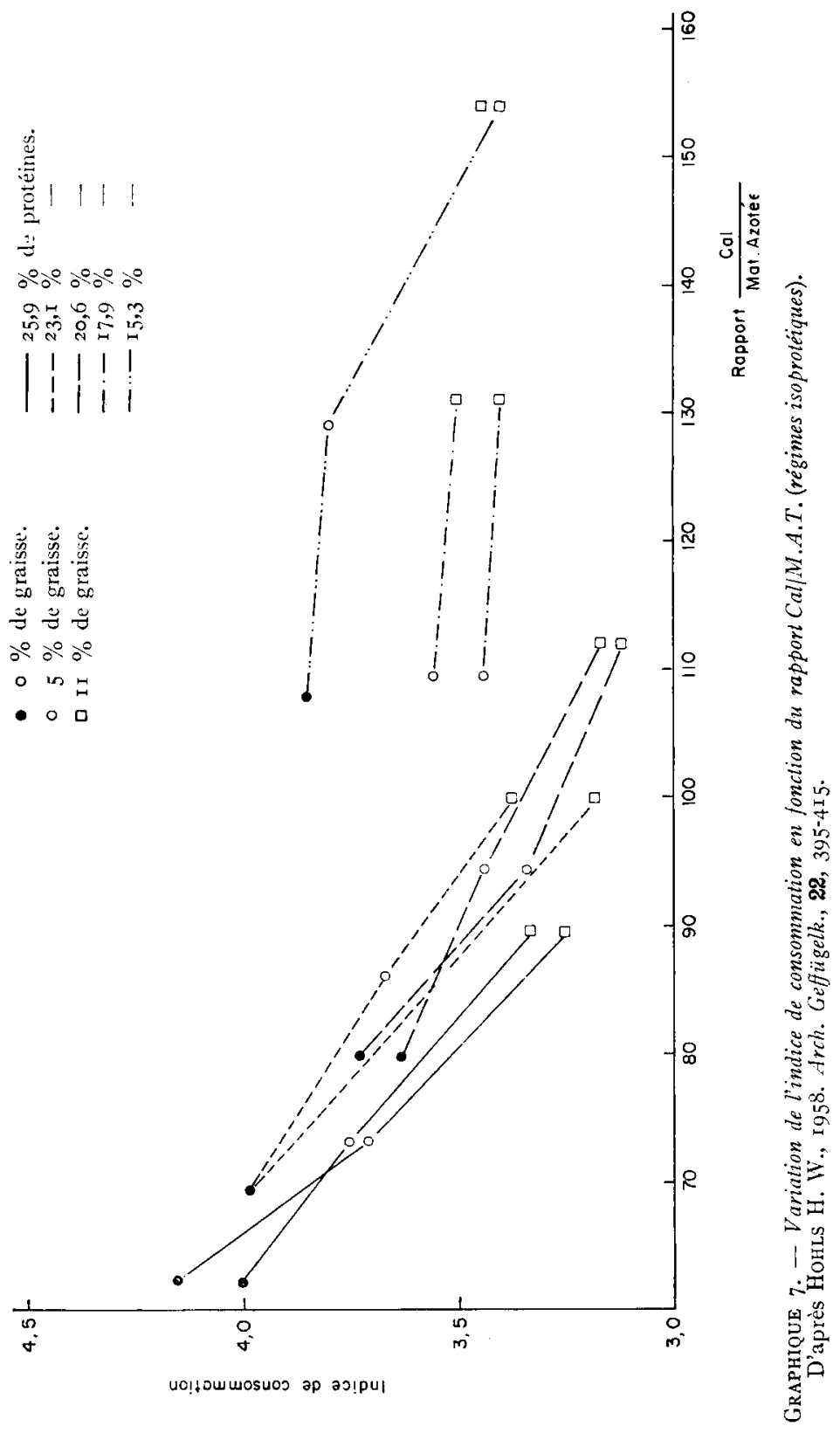




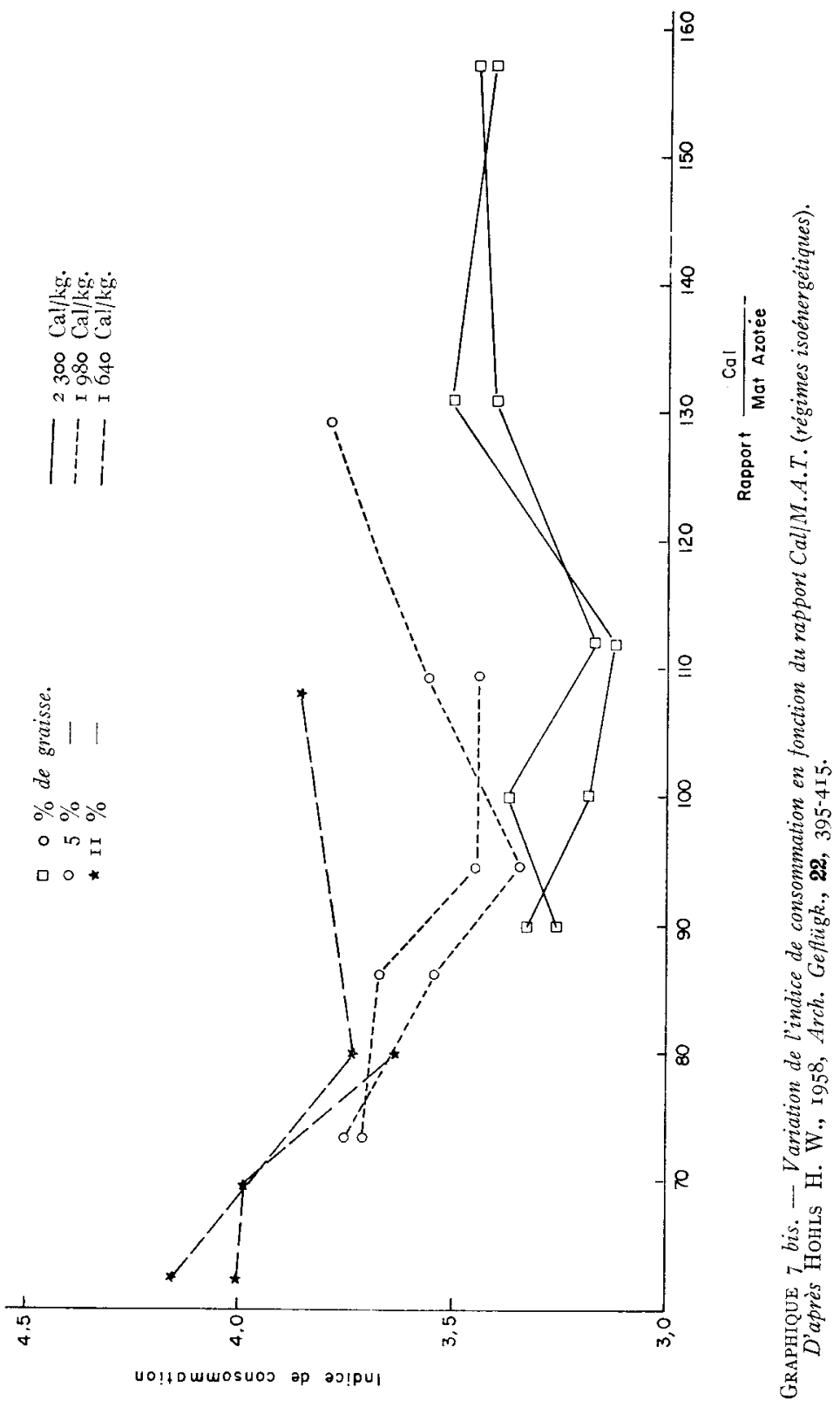


selon qu'il maintient constant le taux énergétique et fait varier le taux protéique ou maintient constant le taux protéique et fait varier le taux énergétique. Dans le premier cas, l'indice de consommation passe par un optimum alors que dans le second, il diminue constamment. Ces résultats confirment ce que nous venons de dire : du point de vue théorique, la variation de l'indice de consommation pour des régimes isoénergétiques se ramène en effet à la quantité de calories ingérées par gramme de gain de poids. C'est la définition de l'efficacité calorique, et HoHLs, ainsi, traduit d'une autre manière des phénomènes identiques à ceux étudiés par les auteurs américains. Un même raisonnement s'applique au cas des matières azotées.

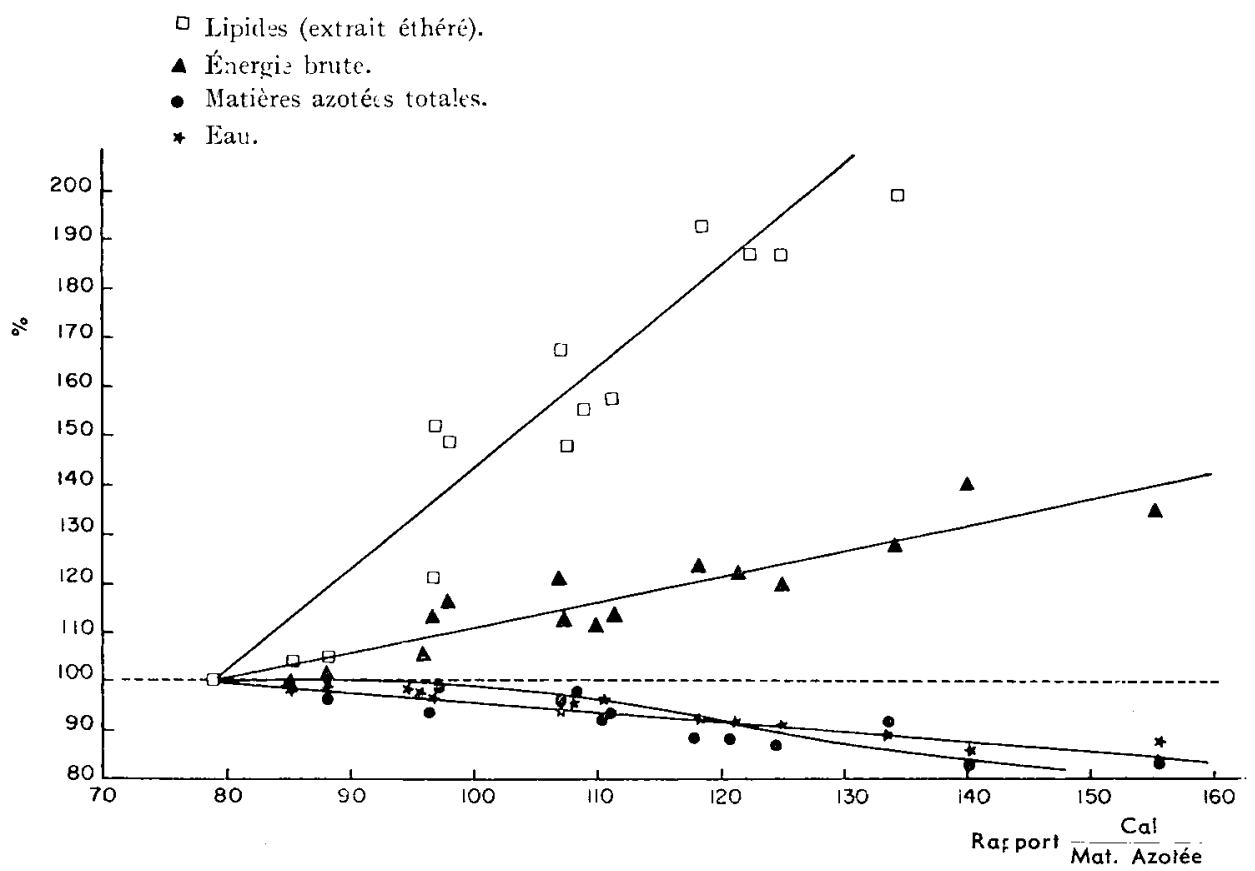

GRAPHicue 8. -- Variation de la composition de la carcasse de lots de poulets ayant ręu des régimes à rapports CallM.A.T. variables.

D'après Donaldson et al. 1956, Poulliry Science, 35, r100-1105.

\section{3. - Infuence du rapport Cal/M.A. sur la composition corporelle}

Les régimes à hauts niveaux énergétiques ont été employés depuis longtemps pour l'engraissement des animaux. L'analyse de carcasses de poulets nourris avec des régimes à rapport $\mathrm{Cal} / \mathrm{M}$. A. ' $\mathrm{T}$. variable doit cependant nous permettre de suivre le phénomène avec plus de précision.

Dès I943, Fraps a noté l'incidence de ce rapport sur l'importance des tissus adipeux, des tissus azotés et de l'eau. Hili et DANsky, (I95I) font des constatations analogues. DONALDSON et al. (I956), à l'aide d'analyses systématiques étudient la variation de la teneur en azote, en lipides et en eau de la carcasse entière en fonction du rapport Cal/M. A. T. (graphique 8). Ce rapport a peu de répercussion sur la teneur en protéines qui passe de 20,3 à I $8 \mathrm{p}$. Ioo du poids du poussin de 4 semaines. Par contre la teneur en lipides passe de 5,6 à I4 p. Ioo et l'eau diminue 
de 70 à $64 \mathrm{p}$. Ioo dans les mêmes conditions. Les chiffres obtenus par LEONG et al. (I959), sur des poussins de 9 semaines dans les expériences citées plus haut, nous ont permis de constater que ces résultats ont une portée générale (graphique 9).

On peut dire que la teneur en matières azotées totales reste relativement constante, tandis que la teneur en graisse augmente dans des limites considérables ( 50 p. IOO). Les variations inverses de la teneur en eau et en lipides, retrouvées dans ces expériences, sont en accord avec la loi générale qui veut qu'un animal qui s'engraisse perde de l'eau.

Ces résultats montrent une fois de plus que le gain de poids est un mauvais critère de la croissance. Pour les rapports élevés alors que l'importance des tissus

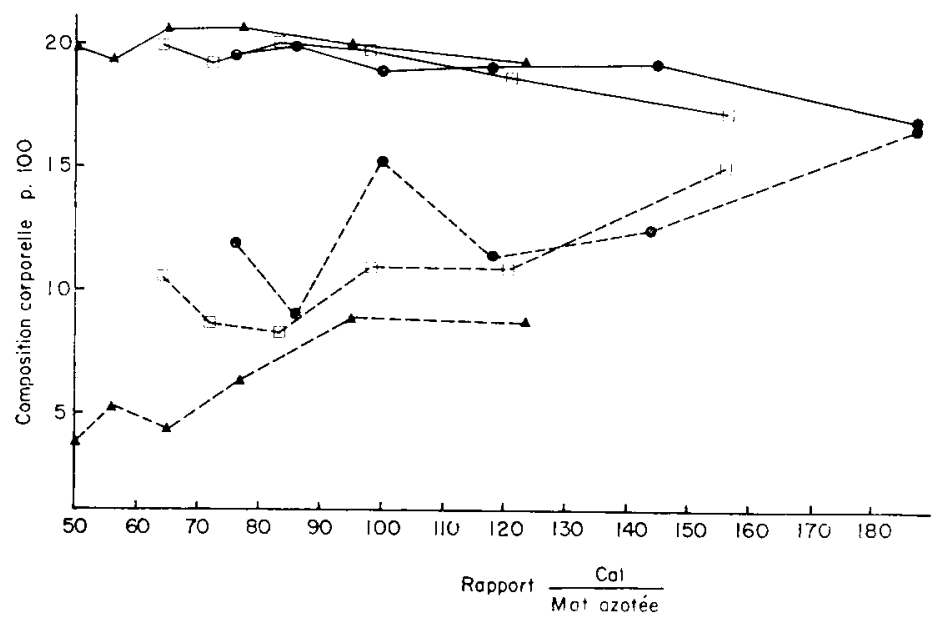

Graphique 9. - Viatiation de la leneur moyenne en proléines et en lipides des carcasses de lots de Poulets avant reçu des régimes à rapports Call M. 1.T. différents.

b'après les données de Leong et al. 1959, Poultry Science, 38 1269-1284, Kxp. I.3.

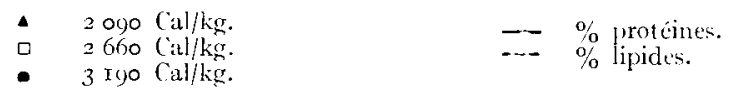

protéiques décroît, celle des tissus adipeux augmente et compense cette diminution. Or, la mesure du poids vif ne donne pas d'indication sur la synthèse des différents tissus. Notons toutefois que DONALDSON et al. (I956) ont montré que quelles que soient les synthèses respectives, le taux de cendres demeure invariable, ce qui peut être interprété comme une constance du développement dı squelette ou de la fixité de la croissance staturale.

Avant 1947 on prenait généralement le taux azoté comme principal critère quantitatif de la valeur d'un aliment. Nous avons signalé que ScotT et al. (I957) cnt voulu prendre en considération le taux calorique. Leurs travaux, et ceux qui ont suivi cet essai, ont finalement dégagé l'importance primordiale du rapport de ces deux taux.

En effet, nous avons vu que les principes azotés et énergétiques d'un aliment pour poussin doivent être apportés dans des proportions déterminées. Si 1'on s'en écarte, la nourriture est mal utilisée, le développement pondéral est ralenti et la synthèse des tissus est modifiée. 
Cependant, nous avons déjà signalé que l'on ne peut définir un rapport Cal/M. A. T. optimal unique (graphique 2 ) - et c'est la variation de cet optimum que nous vous proposons d'examiner en détail.

\section{II. - LES VARIATIONS DU RAPPORT Cal/M. A. T. OPTIMAI,}

Tous les auteurs s'accordent pour dire qu'il existe une zone optimale du rapport Cal/M. A. T. Cependant, ils ne sont ni d'accord sur la valeur moyenne de ce rapport qu'il convient de recommander, ni sur les limites extrêmes au-delà desquelles il convient de ne pas tomber.

Voici par exemple des chiffres pour lesquels les auteurs ont obtenu des croissances optimales.

TABR,EAU I

\begin{tabular}{|c|c|c|}
\hline Auteurs & Jates & Rapport Cal/M.A.T. optimal \\
\hline IRAls . . . . & 1943 & $79-80$ \\
\hline 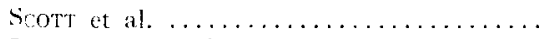 & 1955 & 88 \\
\hline 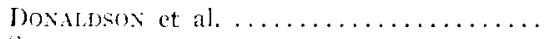 & 1955 & 92 \\
\hline 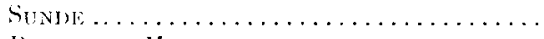 & $1956 a$ & bil \\
\hline 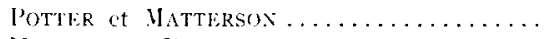 & 1956 & 70 a 123 \\
\hline 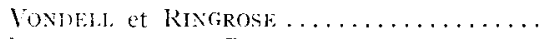 & 1958 & 99 à 11 i \\
\hline BRTLGGRMAN et BARTH ............. & $195 \%$ & $10_{t}^{\prime}$ \\
\hline ('ALET et BARATOY . . . . . . . . . . . & 1961 & 15 a 140 \\
\hline
\end{tabular}

La variabilité, du simple au double, des valeurs du rapport Cal/M. A. T. trouvées ou proposées par les auteurs peut paraître surprenante. En fait, de nombreux facteurs ont une répercussion sur elle. Parmi ceux-ci on connaît 1'âge, le sexe, ou la valeur des matières azotées du régime, etc. D'autres tels que le mode d'élevage ou l'état pathologique ne sont malheureusement que soupçonnés. Nous étudierons les facteurs les mieux connus que nous diviserons en facteurs liés à l'animal d'une part et au régime d'autre part.

\section{A. - FACTEURS itiÉs A L'ANIMAL}

$$
\text { I. - L'âge }
$$

Depuis Heuser (I930), il est recommandé d'employer des régimes différents selon 1'âge de l'animal. En particulier, il convient de diminuer le taux protéique au fur et à mesure que l'animal vieillit. Sans le savoir, on réalisait déjà des régimes à rapport $\mathrm{Cal} / \mathrm{M}$. A. T. de plus en plus fort de la naissance au stade adulte. Dans de nombreux cas, on a cependant utilisé une même gamme de régimes pendant toute la durée de l'expérience. Il est alors assez facile de constater que les lots présentant la plus forte croissance ne sont presque jamais les mêmes au début et à la fin de l'expérience. 
Comme précédemment, on trouve une assez large étendue des valeurs du rapport Ca1/M. A. T. recommandées selon l'âge. Voici par exemple, les chiffres trouvés ou proposés par divers auteurs.

TABLEAU 2

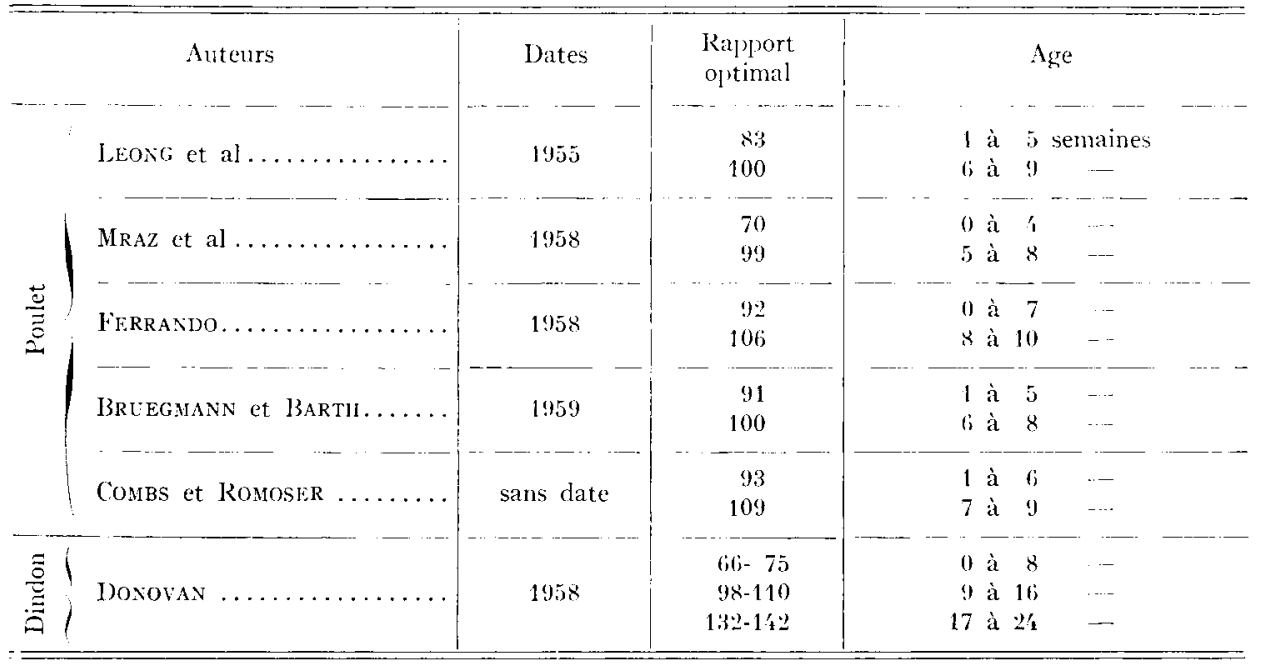

Les données de ScoT'T (I958) citées par WaIBEL (I960) nous ont permis de tracer une courbe de variation du rapport Cal/M. A. T. optimal pour le dindon en fonction de l'âge (graphique ro) : il y a une relation linéaire entre ces deux facteurs.

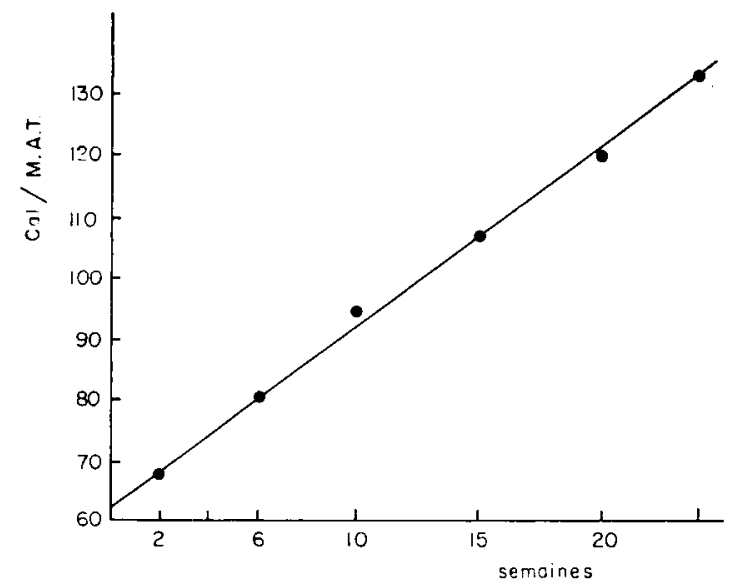

GRAPHIQUE ro. - Variation du rapport Cal/M.A.T. optimal au cours des 24 premières semaines chez le dindon. D'après les valeurs données par:

WaIBEL P., I 960 Feedstuls, 2 (23), 46-52.

\section{Le sexe}

Dans les élevages avicoles, on distribue généralement les mêmes aliments aux femelles et aux mâles en croissance. Cela peut être dû̀ à des nécessités pratiques, mais cela résulte également d'un manque d'information, car on a assez rarement 
comparé l'influence du rapport Cal/M. A. 'T. sur la croissance des poussins des deux sexes.

D'après MFLLEN et HIL, (I954) il n'y aurait pas de différence entre le métabolisme de base des mâles et des femelles, ce qui serait un argument en faveur de la similitude de leurs besoins énergétiques.

Pourtant BruegGmanN et BARTH (I959), signalent que les mâles sont plus sensibles que les femelles à la variation du rapport Cal/M. A. T. sans en déduire de différence de besoin. PEPPER et al. (1953) trouvent que l'enrichissement des régimes avec I ou 2 p. Ioo d'huile de soja améliore la croissance et la finition des femelles alors qu'elle est à peu près sans effet sur les mâles. SHuTzE et a1. (I958) estiment que ceux-ci doivent avoir des besoins azotés supérieurs. De ces deux études, il ressort que les mâles réclameraient un rapport $\mathrm{Cal} / \mathrm{M}$. A. T. plus faible que les femelles. Ce besoin se traduirait au niveau de la composition corporelle. Dansky et HiLL (I952) notent que les femelles déposent davantage de graisse dans leur carcasse que les mâles. HEBER'T et BRUnSON (I957) trouvent également une différence semblable entre les carcasses des animaux des deux sexes. Cette opinion n'est pas partagée par tous. SIEDLER et SCHWEIGERT (I953) trouvent que les coquelets White Rock supportent mieux que les poulettes l'addition de $8 \mathrm{p}$. Ioo de graisse, à condition toutefois qu'elle soit accompagnée d'antibiotique, choline et vitamine $B_{12}$. Cette contradiction avec les études précédentes peut provenir d'une différence de besoins vitaminiques. Mais de plus, elle s'observe sur des mâles White Rock et non sur des New Hampshire.

\section{3. - Origine génétique}

Les contradictions des conclusions précédentes peuvent donc s'expliquer en partie par la diversité des souches employées. Les expériences de comparaison des besoins alimentaires de plusieurs souches ou croisements sont en effets très rares et il est osé de comparer les résultats des essais obtenus avec des souches différentes mais où les protocoles ont été également différents.

LEONG et al. (I959) au cours d'une même série d'expériences sur des animaux de croisement New Hampshire $\times$ Leghorn blanche d'une part, et de souche Arbor Acre $\times$ White Rock, d'autre part, ne constatent que de faibles différences dans la croissance des poussins et concluent que le facteur génétique est négligeable.

Ce résultat ne s'applique pourtant pas à toutes les souches. HoHLs (I960) utilise des Nichols 9/108 et des Leghorn blanches qu'il place dans des conditions nutritionnelles différentes : le taux protéique de ces différents régimes passe de I5 à 28 p. roo et le taux énergétique est modifié par adjonction ou non de matières grasses. Il est constaté que l'amélioration de croissance, résultant de l'élévation du taux énergétique, est maximale pour un taux protéique de 20 p. Ioo chez les Leghorn et de 26 p. roo chez les Nichols. Il y a donc bien une différence de comportement visà-vis de l'équilibre matières azotées totales /énergie chez ces deux souches.

Il ressort de ces études, la nécessité d'élever le rapport Cal/M. A. 'T. avec l'âge de l'animal. Par contre, il est difficile à l'heure actuelle de recommander des normes différentes suivant le sexe et la souche ou le croisement car les expériences en ces domaines demeurent fragmentaires et contradictoires.

Mais si les résultats sont parfois contradictoires et s'il est difficile de se faire une opinion claire de l'ensemble de ces différentes expériences, cela est peut-être dû 
en partie à la diversité des processus expérimentaux. Bien peu d'auteurs ont en effet utilisé des régimes comparables à tous points de vue. F,tant donné les interactions plus ou moins bien connues qui existent entre les différents éléments nutritifs, il faut s'attendre à des effets secondaires sur la croissance, chaque fois que l'on utilise un régime ou un autre.

\section{B - FACTEURS IIÉS AU RÉGIME - Aspect quantitatif -}

Nous avons déjà signalé à propos des courbes du graphique 2 que l'on ne peut définir un rapport Cal/M. A. T. unique, même pour un aliment destiné à un animal donné. Depuis les premiers travaux qui ont montré l'importance de l'équilibre des M. A. 'T. et de l'énergie, plusieurs études ultérieures ont en effet attiré l'attention sur la nécessité de tenir compte aussi de la valeur absolue des termes du rapport Cal/M. A. T.

\section{I. - Infuence de la valeur absolue des termes du rapport sur sa valeur optimale}

a) Cas de la croissance.

LEONG et al. (I955), SUNDE (I956 a) et de nouveau LEONG et al. (I959) font varier à la fois le taux énergétique et protéique du régime afin d'obtenir de nombreux rapports Cal/M. A. T. par toutes les combinaisons possibles des deux facteurs. Nous avons déjà eu l'occasion de rapporter leurs résultats. Toutefois, à partir des chiffres de LEONG et al. (I959) nous avons construit d'abord les courbes de variation du poids vif à 9 semaines en fonction du rapport pour des taux énergétiques différents (graphique 2 ).

Nous obtenons ainsi une famille de courbes qui présentent toutes un maximum différent. Ce graphique illustre donc les conclusions des auteurs qui trouvent un optimum du rapport spécifique de chaque taux énergétique. Si maintenant nous traçons à l'aide des mêmes valeurs les courbes correspondant à chaque taux azoté (graphique 2 bis), nous n'obtenons pas un optimum caractéristique de chacun d'eux, à cause de la trop faible gamme de rapports leur correspondant. Nous l'observons cependant pour les taux moyens; et nous pouvons extrapoler les autres courbes incomplètes sans commettre un gros risque d'erreur et retrouver un rapport optimal différent de ceux que nous observons, puisque PeTERsox et al. (I952) ont démontré leur existence. pour chaque taux azoté. Ce n'est qu'une manière différente de dire que le rapport optimal dépend du niveau énergétique.

Voici groupées dans le tableau 3 les valeurs optimales du rapport Cal/M. A. ' $\Gamma$. publiées par divers auteurs.

TABLEAU 3

\begin{tabular}{|c|c|c|c|}
\hline Auteurs & Taux protéique & Taux énergétique & Rapport optimal \\
\hline \multirow{5}{*}{ DONALDSON et al. $1956 \ldots$} & 19,9 & 1800 & 90 \\
\hline & 22,2 & $\geq 1 / 40$ & $96 i$ \\
\hline & $2 / 1,7$ & 2420 & 96 \\
\hline & 24,7 & 2660 & 107 \\
\hline & 27 & 2097 & $7 s$ \\
\hline \multirow{2}{*}{ LEONG et al. $1959 \ldots \ldots \ldots$} & 29 & 2671 & 92 \\
\hline & 32 & 3200 & 100 \\
\hline \multirow{2}{*}{ REYNTENS $1960 \ldots \ldots \ldots \ldots$} & 20 & 1600 & sio \\
\hline & 21 à 27 & 1000 à 2400 & 90 \\
\hline
\end{tabular}


Ces chiffres mettent en évidence la nécessité pratique d'élever le rapport Cal/M. A. T. toutes les fois que l'on augmente le taux énergétique. Cependant, cette élévation ne devient importante que pour des régimes contenant au moins 2 ooo Cal. productives par $\mathrm{kg}$. De tels régimes hyperénergétiques ne sont obtenus que par adjonction de matières grasses qui, à elles seules, pourraient être une cause au moins partielle de l'augmentation du rapport optimal. Nous reviendrons sur ce point.

L'examen des graphiques 2 et 2 bis appelle une remarque :

Lorsque l'on expérimente avec des rapports voisins de l'optimum permettant la plus forte croissance (autour de Ioo sur le graphique 2 ), on s'aperçoit que la croissance est d'autant meilleure que le taux protéique est plus élevé et que toute augmentation du taux énergétique se traduit par une nouvelle amélioration de la croissance. Cela peut expliquer pourquoi plusieurs auteurs dont POT'TER et al. (I956), SHUTZE et al. (r958) estiment que le rapport Cal/M. A. T. a une faible influence en regard de la valeur absolue des taux azotés et énergétiques.

\section{b) Cas de l'efficacité alimentaire.}

Nous venons de voir que l'influence du rapport Cal/M. A. T. sur la croissance doit se traduire, non pas par une courbe, mais par tune famille de courbes. Nous allons voir qu'il en est de même pour les autres grandeurs influencées par le rapport Cal/M. A. T.

Nous avons signalé que HoHLs (I958) publie les courbes de variation de l'indice de consommation de régimes isocaloriques en fonction du rapport Cal/M. A. ' $\mathrm{T}$. (graphique $7 \mathrm{bis}$ ). On peut en déduire les valeurs optimales suivantes qui sont groupées dans le tableat 4 .

TABLEAU 4

\begin{tabular}{c|c|c}
\hline 1.C & Rapport & Taux énergétique \\
\hline 3,73 & 80 & $1650 \mathrm{cal} / \mathrm{kg}$. \\
$3,3 / \mathrm{k}$ & 94,5 & $1940 \mathrm{-}$ \\
3,12 & 112 & $2300-$ \\
\hline
\end{tabular}

Comme pour la croissance optimale, l'indice de consommation minimal correspond à des valeurs du rapport Ca1/M. A. T. d'autant plus élevées que le taux énergétique est plus fort. D'une manière plus précise les données de LEONG et al. I959, que nous avons utilisées pour la construction des graphiques 4 et 5 nous montrent que les variations de l'efficacité calorique et l'efficacité azotée en fonction du rapport Cal/M. A. T. doivent également être représentées par des familles de courbes, chaque courbe correspondant à un taux énergétique.

\section{c) Cas de la composition corporelle.}

Les diverses données relatives aux analyses de carcasse nous ont permis de suivre la variation des teneurs en eau, matière grasse et matière azotée en fonction du même rapport. Nous voyons (graphique 9) que pour la teneur en lipides, on a une famille de courbes tout à fait comparable aux précédentes; pour les teneurs en matières azotées seulement les résultats sont moins nets, la teneur en protéines étant peu influencée par le rapport Cal/M. A. 'T. et par le taux énergétique. 
En résumé le rapport Cal/M. A. T. n'est pas le seul critère à considéter pour la nutrition énergétique et azotée du poulet. Il faut tenir compte également de la valeur absolue de ses termes puisque le taux énergétique intervient tant sur la valeur du rapport optimal, pour la croissance et l'efficacité alimentaire que sur la composition corporelle.

\section{2. - Rôle direct du taux énergétique}

a) Action sur la croissance.

Si l'on examine la famille des courbes du graphique 2 , on peut voir que les croissances obtenues pour les rapports les plus favorables ne sont pas les mêmes avec les trois courbes. Ce résultat ressort également d'autres études puisque DonaLDson et a1. (I956), trouvent par exemple pour des taux énergétiques de I 800, 2 I40, 2 420, et $2660 \mathrm{Ca} / \mathrm{kg}$ des poids vifs maximaux à quatre semaines, respectivement de : 467 , 475,490 et $472 \mathrm{~g}$.

Le taux énergétique n'aurait donc pas seulement un rôle dans la détermination du rapport optimal, il aurait aussi une influence en so $i$ sur la croissance. Quelque parfait que soit l'équilibre protéines - énergie, il semble qu'une élévation du taux énergétique se traduise par une amélioration de la croissance, jusqu'à une certaine valeur qui serait le taux énergétique optimal. Ainsi peut s'expliquer le succès actuel des aliments enrichis en matières grasses : les régimes courants utilisés jusqu'aux travaux de ScotT et al. (I947) étaient établis sans tenir compte de leur niveau énergétique. Celui-ci se situait vers I 600 à I $800 \mathrm{Cal} / \mathrm{kg}$ et était trop faible pour assurer la meilleure croissance.

L'existence d'un taux énergétique optimal ressort également des études de MRAZ et al. (I957) qui définissent un taux exprimé en calories par unité de volume de 1'aliment. Ils trouvent ainsi que ce rapport ne doit pas s'écarter de la valeur de 0,75 à 0,79 $\mathrm{Ca} / \mathrm{cm}^{3}$, si l'on veut que la meilleure croissance soit maintenue.

\section{b) Interprétation.}

Nous venons de voir que le rapport Cal/M. A. T. ne peut suffire à définir l'équilibre qui doit exister entre les éléments azotés et énergétiques d'un régime, puisque le rapport Cal/M. A. T. doit être d'autant plus élevé que le taux calorique de l'aliment est plus fort.

Cependant, nous avons également vu que les animaux recevant des aliments de même rapport Cal/M. A. 'T., mais de taux énergétique de plus en plus fort, avaient les carcasses les plus grasses. De même si l'on considère, parmi les animaux ayant reçu ces aliments, ceux qui atteignent le même poids à un âge donné, ce seront ceux qui ont reçu à la fois les plus forts taux énergétiques et les plus forts rapports Ca1/M. A. T., qui seront les plus gras.

Nous pouvons donc dire que des aliments, même s'ils ne diffèrent pas par leur effet sur le développement global, diffèrent par leur action sur la croissance de chaque tissu. Chaque taux calorique nécessite un ajustement déterminé au taux azoté pour que la meilleure efficacité alimentaire soit assurée, mais il entraîne aussi une synthèse tissulaire déterminée.

Nous avons rapproché ces résultats des travaux de HiL, et DANSKy (I954) sur les mécanismes d'ingestion des aliments par le poulet. Nous n'entreprendrons pas 
une revue des nombreuses études faites sur l'appétence, ce qui déborderait largement le cadre de cet article.

Signalons cependant que KARE et al (I957) a montré que le poussin avait un certain sens du gồt et que le déterminisme de la consommation du poulet était complexe. Cependant d'autres études ont montré que, toutes choses égales par ailleurs, la composition globale du régime avait une grande influence sur elle. Ainsi les taux caloriques et azotés semblent jouer un grand rôle.

Les premiers auteurs PETERSON et al. (I952), Williams et GraU (I953) ont admis avec HrL, et DANSKY (I950), que le poussin cherche d'abord et avant tout à satisfaire son besoin énergétique. Des études plus poussées de ces derniers auteurs (r954) ont montré que grosso-modo le poussin consomme bien une quantité d'énergie proportionnelle à son métabolisme de base. On trouve une proportionnalité entre la consommation de calories et le poids vif élevé à la puissance 0,76 .

Lorsqu'on fait varier seulement le taux protéique du régime, on n'observe pas d'influence directe sur la consommation. Ce taux aurait par contre, une influence indirecte en modifiant la croissance de l'animal et, par conséquent, son besoin énergétique et sa consommation d'énergie. Ce ne serait pas lui qui déterminerait l'appétit, même si l'apport de protéine du régime ne couvre pas le besoin azoté de l'animal. Afin d'assurer la couverture du besoin azoté, il convient donc de maintenir dans le régime une relation très précise entre les matières azotées et les calories.

Cette théorie ne donne pas entière satisfaction. Les auteurs eux-même, HIL L et DANsky (I954), s'aperçoivent qu'elle n'est pas vraie pour des régimes où le taux énergétique est inférieur à I $500 \mathrm{cal}$. productives $\mathrm{kg}$, ou supérieur à $2200 \mathrm{cal}$. productives par $\mathrm{kg}$ environ. Lans le premier cas, le poussin ne peut consommer suffisamment d'aliments pour compenser intégralement le déficit calorique de sa ration. Dans le second cas, bien qu'il réduise le niveau de ses ingérés, il consomme un excès de calories.

I ans la mesure oì le poussin dispose d'un aliment à taux calorique trop élevé, il ingère donc trop de calories et cet excès coïncide avec 1'accroissement de la synthèse de tissus adipeux. Nous avons en effet vu que le rapport des synthèses tissus adipeux s'élève au fur et à mesure que le rapport Cal/M. A. T. croît, et, à rapport constant, quand le taux énergétique croît.

Autrement dit, la synthèse des tissus adipeux est liée bien davantage au taux énergétique du régime qu'au rapport Cal/M. A. T., comme l'avaient signalé L_tong et a1. (I959); et ceci semble dû au fait que le poussin nourri ad libitum ingère d'autant plus de calories que l'aliment en contient davantage, ce qui peut paraitre évident à première vue et qui n'est pourtant valable que pour des taux énergétiques très bas ou très élevés.

\section{c) Conclusion.}

Lorsque nous disons qu'il existe un rapport optimal pour le développement, correspondant à chaque taux calorique, nous ne faisons que simplifier les faits: nous restreignons la signification physiologique du développement, puisqu'il s'agit en fait d'un phénomène global recouvrant une multitude de développements tissulaires particuliers. Et chaque rapport optimal, ainsi considéré, entraîne en fait, une croissance particulière. 


\section{ASPECT QUALITATI}

\section{I $^{\circ}$ Rôle de la nature des matiires azotées}

Jusqu'à ces dernières années, aucun travail n'a été entrepris à notre connaissance dans le but de démontrer le rôle de la nature des protéines sur la valeur optimale du rapport Cal/M. A. T.

Toutefois, dans la masse des expériences qui concernent la détermination de cette valeur, il est possible de dégager ce rôle en collationnant plusieurs séries de résultats.

Au hasard de leurs essais, Wir.LIAMs et GRAU (I953) remarquent que les taux énergétiques les plus faibles provoquent la meilleure croissance lorsque le régime de base contient du tourteau de sésame, c'est-à-dire une protéine déficiente en lysine. A l'inverse, LEONG et al. (I959), constatent que les poussins supportent mieux les taux énergétiques élevés, chaque fois que le régime est enrichi en méthionine et en créatine, cette dernière étant utilisée afin de pourvoir aux besoins en arginine de l'animal. Ils peuvent ainsi obtenir pour le taux énergétique de $2670 \mathrm{Cal} / \mathrm{kg}$ une croissance optimale avec un rapport Cal/M. A. T. de I20 au lieu de 92 quand la protéinə n'était pas supplémentée. C'est dans le même esprit que Combs (I959), suggère dans une conférence de vulgarisation que l'on pourrait vraisemblablement élever le rapport Cal/M. A. 'T. au-dessus des normes qu'il cite à la condition d'employer des régimes mieux équilibrés en acides aminés.

La nature des protéines retentit sur les besoins énergétiques et azotés de l'animal. A l'inverse, on pouvait donc s'attendre à ce que le niveau énergétique des ingesta influence le besoin en acide aminé.

I) fait, RINGroste et al. (I95.5) cherchent à satisfaire le besoin en méthionine en distribuant au poussin des doses croissantes d'hydroxy-analogues de la méthionine. Ils trouvent que ce besoin dépend des taux azoté et énergétique du régime mais d'une façon bien plus nette, du rapport des deux. Pour Twining et al. (I955) ce besoin exprimé en pourcentage de matières azotées totales, diminue légèrement quand le taux protéique augmente, croît légèrement quand le taux calorique augmente. On comprend alors l'évolution rapide du besoin en méthionine sous l'effet du rapport que trouvent les auteurs précédents. Les études détaillées de RosENBERG et BALIDIN (I957) confirment les résultats précédents et montrent qu'il y a une relation linéaire entre le taux énergétique du régime et le taux de cet acide aminé exprimé en pourcentage de la ration.

Cela prouve que le rapport Cal/M. A T. optimal dépend de la composition des matières azotées totales en un acide aminé : la méthionine. RoSENBERG et BALDINI supposent cependant que ce résultat s'applique également aux autres acides aminés indispensables.

Il y a un certain temps déjà, Almovis's (I953) a établi que le besoin en acides aminés exprimé en pourcentage des matières azotées totales diminue lorsque le taux protéique augmente. Malheureusement, ces études ont été poursuivies sans tenir compte du taux énergétique des régimes employés. Il est cependant probable qu'il s'agissait de régimes à peu près isoénergétiques donc à rapport Cal/M. A. 'T. décroissants. De ce fait, GORDON et al. (I $95^{8}$ ) déclarent que lorsqu'on prend soin de maintenir le rapport Cal/M. A. T. constant, la norme en acides aminés ne dépend plus du taux protéiques. Ils conseillent d'appliquer les normes en acides aminés proposées par le N. R. C. uniquement lorsque l'on utilise des régimes classiques (taux protéique de 
20 p. Ioo et taux énergétique de I 800 cal. productives). Par contre, pour des rapports Cal/M. A. T. plus élevés, ces normes ne sont plus valables.

I)ans le même ordre d'idées, COLEOU, cité par FERRANDo et Col EOU (I g60), fournit au poussin en finition des régimes blé-lait écrémé dans lesquels le rapport Cal/M. A. T est de $\mathrm{I} 30$. Les croissances obtenues sont bonnes. Elles demeurent identiques lorsque le rapport est amené à IO5 - IIo, par adjonction de tourteau de soja. Il conclut que les " normes établies avec des régimes à dominante soja-maïs sont susceptibles de varier avec le type d'aliment et notamment en fonction de la qualité de la protéine ".

L'ensemble de ces résultats semble pronver que le rapport Cal/M. A. T. optimal doit être adapté à la valeur biologique de la protéine alimentaire.

C'est pour le démonter que CALET et al. (I96I) ont entrepris une série d'expérience oì ils mesurent la consommation spontanée d'énergie lorsque le poussin reçoit simultanément dans deux mangeoires séparées les substances ternaires et les substances azotées, ces dernières, d'origine variable étant allouées en quantité constante. Ils comparent les résultats à ceux fournis par des poussins qui reçoivent la même quantité de matières azotées en mélange avec les substances énergétiques.

Ils observent une amélioration de la croissance et de la consommation d'énergie avec la farine de poisson, lorsqu'on laisse au poussin le libre choix de ses calories. A l'opposé avec le tourteau d'arachide, la consommation spontanée d'énergie s'abaisse de 30 p. Ioo sans répercussion fâcheuse pour la croissance. Il en résulte que la présentation d'un régime en mélange où le rapport Cal/M. A. 'T. est donné, se traduit par un excès d'énergie ingérée pour les protides de faible valeur et par un défaut pour les protides de haute valeur.

Ces résultats ont une portée plus générale et s'étendent à d'autres espèces animales. AmRAham et a1. (I06I) distribuent à des rats blancs et à des poussins deux repas séparés selon le protocole précédemment décrit. Le premier repas ne contient que des substances azotées dont les auteurs font varier largement la nature : farine de poisson, caséine enrichie en méthionine, tourteau de soja cuit, tourteau d'arachide et gluten de blé. Leurs résultats montrent que 1'on peut classer les différents protides avec la même hiérarchie, aussi hien au moyen de leur valeur biologique que de la consommation spontanée d'énergie qu'ils provoquent.

Notons également que dans tous les cas l'efficacité azotée des protides alimentaires est la plus élevée lorsque l'animal choisit lui-même la quantité qui convient à leur utilisation métabolique.

La consommation spontanée d'énergie et le rapport Cal/M. A. T. déterminés par le libre choix du poussin dépend donc de la nature de la protéine. Des expériences ultérieures de CALET et BARATOU (I96I) montrent que ces rapports, déterminés par le poussin lui-même, sont respectivement de :

95 pour le tourteau d'arachide;

I25 pour le tourteau de soja;

I 40 pour la farine de poisson.

Ces résultats sont épaulés par des observations de BRETTĹ et al. (Ig60) qui définissent auparavant l'équivalent ouf des matières azotées. L'équivalent cuf est la quantité de protéines d'œuf exprimée en grammes qui possède la même efficacité que roo $g$ de protéine considérée. I'équivalent œuf d'une protéine n'est pas autre chose que sa valeur biologique relative lorsque l'on prend celle de l'œuf égale à roo, c'est-à-dire l'index chimique de MiTCHEL, et BLOCK. Jans ces conditions les auteurs 
observent que le rapport entre l'équivalent œuf et les calories productives du régime doit être constant et égal à $0,05^{8}$ poirr assurer la meilleure croissance, ce qui correspond à un rapport Cal/M. A. T. de I72 pour les protéines de l'œuf. Il convient de noter que ces valeurs ne sont valables que pour des taux énergétiques variant dans des limites très étroites de I 800 à 2000 calories productives par kg d'aliment.

La notion " d'équivalent œuf " ne fait intervenir que la composition en acides aminés indispensables de la protéine en attribuant seulement un rôle énergétique aux autres formes azotées. Elle ne tient pas compte non plus du mode de présentation des acides aminés.

On sait en effet, depuis ROSENBERG et a1. (r954), que le besoin énergétique du rat est considérablement augmenté lorsque l'on fournit la même quantité d'acides aminés sous forme libre au lieu de les distribuer engagés dans des combinaisons prótidiques. Plus récemment, LEVEILLE et Fisher (I958) déterminent à l'aide d'acides aminés libres le besoin en acides aminés du coq adulte et constatent que pour y parvenir, il faut élever de 20 p. Ioo le niveau calorique de la ration.

D'autres formes d'azote soluble ont été étudiées chez les espèces domestiques supérieures. Nous les avons laissées sous silence, étant donné leur faible importance en alimentation des volailles.

Dans le choix du taux énergétique optimal du régime, il ne suffit donc pas de prendre en considération la quantité de matière azotée totale qu'il contient ; il y a lieu de tenir compte dans sa détermination, de la nature et de la qualité des protides du régime. Il y a compensation de la quantité de matières azotées par leur qualité.

\section{2. - Rôle de la nature des matières énergétiques}

\section{a) Position du problème.}

Il est difficile à l'heure actuelle de se faire une idée claire du rôle de l'origine des calories dans la nutrition du poulet.

Un premier groupe d'auteurs attribue au poulet une aptitude marquée à bien supporter les calories grasses. DONALDSON et al. (I956) constituent des régimes à taux protéique variable dans lesquels ils ajoutent $0,7,5$ et I $_{5}$ p. Ioo de suif. Lorsque le taux énergétique s'élève, ils observent que le rapport Cal/M. A. 'T. maximal toléré par l'animal est d'autant plus fort que la proportion de calories grasses est plus élevée. La composition corporelle de l'animal est également modifiée : la valeur en lipides des carcasses augmente avec celle des matières grasses dı régime.

Bien que ces auteurs concluent à une certaine supériorité des calories lipidiques sur les calories glucidiques, il convient de faire remarquer que dans leur expérience, deux facteurs peuvent être à la fois responsable des phénomènes observés : en augmentant le taux de matière grasse du régime, ils élèvent parallèlement son taux énergétique et nous avons vu l'importance de ce taux sur la détermination du rapport Cal/M. A. 'I' optimal.

BALDINI et ROSENBERG (I957) proposent une expérimentation plus précise. A chaque taux énergétique du régime, ils comparent 1'efficacité des calories lipidiques et glucidiques en apportant ro p. Ioo de calories totales de la ration, soit par de 1'amidon, soit par différentes matières grasses. Ils concluent formellement qu'à partir du moment où le besoin en acides gras essentiels est couvert, les lipides possèdent les 
mêmes propriétés sur la croissance que les glucides. Ils n'observent pas non plus de répercussions de l'origine des graisses sur la croissance.

Devant la contradiction soulevée par les deux séries d'expériences, un troisième groupe d'étude propose une explication plus nuancée des faits.

Tout d'abord, LEONG et al. (I959) aboutissent aux mêmes résultats que DONAI,ISON en opérant de la même manière mais leur interprétation est différente. Contrairement à ce dernier, ils attribuent l'effet positif des graisses sur la croissance, beaucoup plus à l'élévation du taux énergétique du régime qu'à leur nature propre. En ce qui regarde la composition corporelle, par contre, ils estiment que la nature des calories retentit sur la teneur lipidique des carcasses.

C'est à peu près aux mêmes conclusions que BARTH arrive en I959. Il prépare un mélange ( $75 \mathrm{p}$. Ioo blé et $25 \mathrm{p}$. Ioo suif) qui possède la même valeur énergétique que le maïs. Puis il substitue ce mélange au suif dans l'aliment complet du lot témoin en vue de réaliser des régimes isoénergétiques. En remplaçant progressivement les 6o p. roo du maïs du régime de base par des quantités égales du mélange précédent, il retrouve les résultats de BALDINI et ROSENBERG : les calories ont la même efficacité pour la croissance quelle que soit leur origine. Par contre, pour la composition corporelle, il est en accord avec L,EONG et al. (I959). Plus le régime est riche en matières grasses, plus la carcasse est chargée en lipides. Il n'ose pas cependant conclure à une différence réelle d'efficacité des lipides et des glucides, car il n'est pas sûr que la valeur énergétique qu'il a attribuée aux matières grasses expérimentées soit exacte.

Il convient de noter au passage que les régimes entichis en suif produisent à la fois un développement accru de tissu adipeux et une stéatose hépatique.

\section{b) Incertitude des mesures de l'énergie productive des matières grasses.}

Une grande difficulté réside en effet dans le fait qu'on ne connait pas toujours avec certitude la valeur énergétique des différentes matières grasses. On a recours pour l'estimation de ces valeurs à des tables qui sont, soit anciennes, soit incomplètes, soit contradictoires. On sait en effet que le nombre de calories productives par kg de matières grasses d'origine animale varie considérablement selon les auteurs. Il est de 4620 pour FraI's (I946) et de 6380 pour HILL (I954) (valeurs citées par Donaldoson et a1. I956).

Nous insistons ici sur le fait que nous avons préféré les calories productives de Fraps comme unité d'énergie, uniquement parce qu'elles ont été utilisées dans la plupart des études que nous avons consultées. Il n'empêche que plusieurs auteurs : Hill et ANDERSON ( $195^{8}$ ) ont montré que l'emploi de cette unité aboutissait à des erreurs plus grandes, que celui de la calorie métabolisable. Cette erreur serait particulièrement importante dans le cas des graisses. (BIELY et MARCH, I957).

La détermination du nombre de calories productives/kg de matières grasses dépend en effet de leur taux de digestibilité. A son tour, 1'absorption intestinale des graisses dépend de leur nature propre, mais également du reste de la ration qui interfère sur elle.

a) Facteurs intrinsèques : Digestibilité des graisses sensu stricto. - La digestibilité des lipides est un phénomène encore mal connu et essentiellement variable. L'absorption intestinale semble dépendre du taux de matière grasse, de son point de fusion, du degré d'estérification des acides gras, de leur nature et de leur proportion. 


\section{Influence du taux de matière grasse}

D'après DuckworTh et al. (I950), la digestibilité des graisses diminue chez le poulet, lorsque leur taux dépasse 6 p. Ioo dans le régime. Elle diminue également au fur et à mesure que leur taux alimentaire augmente. Cette observation n'est possible que pendant les premières semaines d'expérience. Au-dèlà, la digestibilité des régimes très riches en matières grasses s'améliore avec le temps. Il faut que le poussin s'adapte à son nouveau régime et cette adaptation est d'autant plus facile que la graisse est plus désaturée.

'Toutefois, plus récemment, WILLIAMs et al. (I959) attirent l'attention sur la prudence qu'il faut manifester dans l'interprétation des coefficients de digestibilité des lipides. A l'inverse des précédents auteurs, ils observent que le coefficient de digestibilité apparent des graisses végétales est amélioré lorsque le taux de lipides du régime augmente. Il demeure cependant constant lorsque l'on corrige les résultats de la quantité de lipides excrétée par l'animal qui reçoit le régime de base seul.

\section{Inffuence de la nature des matieres grasses}

Plusieurs auteurs ont trouvé que les graisses d'origine végétale étaient plus digestibles que les graisses d'origine animale. Il y a cependant des contradictions. WILI IAMS et al. sont en accord avec cette idée pour la plupart des lipides sauf pour la graisse du poulet qui est la plus digestible.

En fait, Biely et March (I954), puis Sunde (I956 b) ont comparé plusieurs graisses d'origine animale et végétale à des graisses hydrogénées et des acides gras libres. C'est la nature des acides gras qui retentit sur leur digestibilité. L,es lipides les plus désaturés sont les plus digestibles. Les auteurs concluent à l'incapacité des acides gras à longue chaîne carbonée d'être utilisés par le poulet, et à l'inaptitude des graisses hydrogénées qui ne sont digestibles qu'à 40 p. Ioo environ.

Il convient ici de signaler les résultats de CARROLL (I959) qui observe chez le rat une réduction de croissance sous l'effet des huiles hydrogénées. Cet effet disparait toutefois dès que l'on ajoute au régime des huiles désaturées ou du linoléate de méthyle.

D'une manière plus générale, il ne faut pas oublier que les graisses alimentaires peuvent subir de profondes modifications, soit au cours de leur préparation industrielle, soit spontanément au cours de leur conservation. La présence d'acides gras atypiques, acides oxydés, isomères, polymères, etc. a nécessairement un retentissement plus ou moins grand sur la valeur nutritionnelle des graisses (RAvLIN et al. I959).

\section{Infuence du point de fusion}

On a voulu attribuer le fait, que les graisses les plus désaturées sont les plus digestibles, à leur point de fusion très bas. Cependant le résultat n'est valable que pour des graisses dont le point de fusion est supérieur à $46^{\circ} \mathrm{C}$. Au-dessous de cette valeur, la digestibilité est pratiquement complète. Ces résultats ont été confirmés récemment par RENNER et HILL, (I958).

\section{Infuence du degré d'estérifucation}

Lorsque l'on fournit les mêmes acides gras sous formes libre ou estérifiée, leur digestibilité est inégale. Ce sont les acides gras sous forme de glycérides qui sont les 
plus digestibles (CARrol, et Richards, I958). De toute façon la digestibilité est faible, si les acides gras sont à longue chaîne.

ß) Facteurs extrinsèques. - Parmi ces facteurs, celui qui nous intéresse directement concerne le taux protéique.

Chez le rat, BARnes et al. d'une part, et Forises et al. (I946) de l'autre, énoncent que la digestibilité est améliorée par la présence de protides dans le régime. BIELY et March (I957), chez le poulet, sont également en accord avec les résultats précédents à la condition que l'on expérimente avec des taux protéiques élevés. Ils complètent leurs données en démontrant le comportement différent des graisses selon leur nature Ainsi ce sont les graisses les moins digestibles qui sont le plus valorisées par les taux protéiques élevés.

Nous pouvons donc déjà dire que la détermination de la valeur énergétique des graisses est extrêmement délicate au moyen de calories productives. Cette difficulté, à elle seule, rend les comparaisons de l'efficacité des calories grasses et des calories glucidiques fort discutables. Mais d'autres causes compliquent le problème.

c) Rôle des constituants non énergétiques des matières grasses.

Il ne faut pas considérer simplement les graisses comme des sources d'énergie puisqu'elles apportent un certain nombre de constituants directs de l'organisme et de métabolites: Phosphatides, stérols, vitamines liposolubles et surtout acides gras essentiels. Les expériences de REISER et CovCH (r949), montrent il est vrai, que les besoins en ces acides sont faibles. De même SUNDE (I956 b) trouve que les acides oléiques, linoléiques, linoléniques ajoutés à un régime équilibré normal sont sans effet, car le régime à base de céréales fournit une quantité amplement suffisante d'acide gras essentiels. Davis avait montré, dès I94I, que 4 p. Ioo de lipides totaux suffisaient largement pour couvrir les besoins.

Mais les lipides peuvent aussi agir par les nombreux corps autres que les acides gras et glycérides qu'ils renferment. Dans une étude précédemment citée, BaLnini et ROSLNBERG (I957) pensent que tous ces corps possibles n'ont aucune influence et que les lipides n'agissent que par leurs calories. Le pouvoir énergétique ne suffit pourtant peut-être pas pour expliquer les résultats obtenus par PEPPER et a1. (I953). Ces auteurs observent en effet une même amélioration de croissance en remplaçant I ou 2 p. Ioo de maïs du régime par la même quantité d'huile de soja. Ils font l'hypothèse que d'autres facteurs que l'énergie pourraient intervenir.

Chez le rat tout au moins, CAI,ET, FRANÇOIS et JACQUOT (I957), montrent que 2 p. Ioo de lécithine améliorent de $19 \mathrm{p}$. Ioo le gain de poids. On sait d'autre part, que la composition en vitamines liposolubles des graisses varie considérablement selon leur origine : une huile de maïs non raffnée, par exemple peut apporter des doses de l'ordre de $0,20 \mathrm{p}$. Ioo de vitamine E. Une telle dose peut modifier largement l'efficacité du régime.

Ici encore, nous nous heurtons donc à une nouvelle difficulté dans l'emploi des graisses, puisqu'une addition de lipides peut entrainer un effet positif important sur la croissance, dû̀ à une cause toute autre que l'apport de calories.

d) Différences de rôle métabolique entre glucides et lipides.

Une troisième difficulté réside dans l'utilisation des sucres et des acides gras par 1'organisme. Nous avons signalé que certains auteurs tels que ForBES (I946) et LEONG 
et al. (I959) pensent que les lipides auraient une action favorable directe sur la lipogenèse. Tout se passe en effet comme si les tissus adipeux étaient plus rapidement re-synthétisés à partir d'acides gras que de glucides. Nous n'avons cependant trouvé aucune étude récente se rapportant à ce sujet, effectuée sur le poulet.

Mais nous pouvons signaler les résultats de Kropotowski et SmiETANSKA (I960) qui étudient le métabolisme lipidique du rat à l'aide d'acétate marqué au $\mathrm{C}^{14}$. Ils démontrent que la lipogenèse hépatique est accrue par adjontion d'huile de soja dans le régime.

Mais de même, qu'il a été démontré qu'il existait des interactions lipides - protides, au niveau de la digestibilité, de même au niveau du métabolisme intermédiaire, il existe des interactions glucides-protides et lipides-protides. TERroINe, cité par RAULIN (I958), en accord avec de nombreux chercheurs, a démontré que les glucides ont un effet d'épargne vis-à-vis des protides alimentaires que ne possèdent pas les lipides. Cette opinion n'est cependant pas partagée par tous. Des expériences plus récentes ont abouti au résultat opposé : les lipides permettraient une rétention azotée plus forte. (Forbes et al, I946). Enfin, d'autres auteurs (Thompson et MonRo, I955), (Yoshida et al., I957) ne trouvent aucune supériorité des glucides sur les lipides. Selon Swanson (r958), il s'agirait de deux mécanismes différents: Les glucides auraient un effet spécifique d'économie des protides, à condition d'être ingérés à peu près en même temps que ceux-ci ; les lipides agiraient en retardant le catabolisme azoté chez les animaux carencés. Cet effet d'épargne serait variable selon les graisses et serait dû en grande partie à l'insaponifiable.

En conclusion, nous pouvons dire que l'emploi des graisses en tant que source énergétique doit toujours être effectué avec la plus grande prudence :

I. - Il semble en effet que l'estimation de leur valeur énergétique au moyen de calories productives, sans tenir compte du reste de la ration, soit en général délicate.

2. - A cette imprécision s'ajoutent des effets secondaires dus à certains composants des lipides eux-mêmes, et non aux calories qu'ils apportent. Ces effets peuvent fausser apparemment la valeur énergétique des matières grasses.

3. - Einfin, lorsque nous définissons la norme énergétique en fonction de norme azotée, il faut tenir compte des interactions existant entre elles et qui, pourtant sont difficiles à prévoir.

Il est donc impossible de définir correctement un besoin énergétique sans préciser par quels éléments il est assuré.

Toutes ces difficultés font de plus qu'il est impossible d'affirmer que l'apport des calories sous forme de lipides au lieu de glucides impose une augmentation du rapport optimal Ca1/M. A. T. Il est également impossible d'affirmer que les lipides alimentaires retentissent directement sur la lipogenèse du poulet, bien que cela paraisse vraisemblable.

\section{3. - Discussion}

Nous venons de voir la complexité des variations du rapport Cal/M. A. T. et les problèmes que posent ces variations.

En réalité, ce rapport ne fait qu'exprimer la relation de deux besoins. Or, pour déterminer le besoin d'un animal en un élément nutritif, on réalise généralement une série de rations contenant des doses croissantes de cet élément, et on note celle au- 
delà de laquelle la croissance n'est plus améliorée. On a procédé de même pour le rapport Cal/M. A. T., mais ici le problème se complique du fait que 1'on distribue l'aliment ad libitum.

Si le poussin mangeait pour satisfaire son besoin en un seul élément, l'énergie par exemple, comme 1'avaient pensé tout d'abord, Hil L et DANSky (I954), la détermination du rapport $\mathrm{Cal} / \mathrm{M}$. A. T. optimal serait un moyen commode de mesurer le besoin du second élément. Fn fait, nous avons vu que les choses sont beaucoup plus complexes: On ne peut savoir, a priori, quelle quantité d'un aliment de composition commune, le poussin consommera.

Lorsqu'avec une collection, forcément limitée de régimes, on constate que l'un d'eux permet un développement meilleur que les autres, on admet qu'il assure les besoins en matières azotées et en énergie de 1'animal. Or en réalité, on ne sait si l'un de ces besoins, voire chacun d'eux, n'est pas couvert par excès. On ne sait par exemple, si une partie des matières azotées n'est pas dégradée à des fins purement énergétiques.

D’une manière plus générale, dans toutes les expériences où le régime est constitué par un aliment complet, on impose à l'animal, la nécessité d'ingérer tous les éléments nutritifs dans des rapports déterminés et qui ne sont pas forcément adéquats. Or les éléments autres que ceux dont on veut étudier le rôle peuvent avoir une action directe ou en retour sur l'ingestion de nourriture. La détermination exacte des besoins azotés et énergétiques est donc extrêmement délicate par cette méthode.

Si l'on considère la détermination du rapport Cal/M. A. T. optimal comme la résultante de la détermination des besoins énergétiques et protéiques, une autre objection apparait. On ne peut en effet discerner le besoin quantitatif de chacun des éléments de leurs besoins qualitatifs.

En effet, lorsque l'on veut augmenter dans des régimes le taux protéique tout en maintenant constant le taux énergétique, il faut faire appel pratiquement à des doses croissantes de tourteau ou de farine animale. Il en résulte une modification de la valeur biologique de l'ensemble des protéines du régime et l'on ajoute à la variation escomptée de la quantité, une modification de la nature des matières azotées.

Certains auteurs, tels que Donaldoson et al. (1956) ont essayé d'y remédier en ajustant les proportions de tourteau et de farine animale, et en les supplémentant éventuellement avec des acides aminés libres, de manière à conserver une même valeur biologique. Mais ils se heurtent alors à l'objection de certains auteurs (ALMQUist, I953), qui, ayant travaillé avec des régimes sensiblement isoénergétiques, ont trouvé que le besoin en acides aminés exprimé en pourcentage de la protéine, varie en sens inverse du taux azoté. Le maintien d'un même équilibre entre les acides aminés dans la protéine, n'est donc pas recommandé puisqu'il ne respecte pas la variation du besoin en acides aminés en fonction du taux azoté.

De même, si l'on utilise une protéine de nature constante en proportion également fixée dans des régimes de plus en plus énergétiques, on ne respecte pas l'augmentation du besoin en acides aminés mise en évidence par BALDINI et ROSENBERG (I955), d'autre part.

La distribution d'un aliment complet où la proportion des constituants est fixée à l'avance, impose à l'animal l'ingestion de quantités proportionnelles de protéines, d'acides aminés et de substances énergétiques minérales et vitaminiques. Or, le besoin en acides aminés varie avec chacun des termes du rapport Cal/M. A. ' $\mathrm{T}$. Lorsque, comme la plupart des auteurs l'on fait, on choisit à la fois des taux protéiques et 
des taux énergétiques variables dans le but d'obtenir une multitude de rapports Ca1/M. A. T., la probabilité d'assurer au plus juste le besoin en acides aminés de l'animal est extrêmement réduite. On a cru faire varier deux facteurs à la fois, mais on a en fait entraîné d'autres variations qu'il n'est plus possible de contrôler.

De ceci, nous devons done conclure qu'il est impossible de définir un rapport Cal/M. A. 'T. optimal où les M. A. T. seraient apportées par une protéine idéale. C'est-à-dire qu'il est impossible de définir à la fois le besoin énergétique, le besoin quantitatif et le besoin qualitatif de M. A. 'T. Nous devons nous contenter de définir

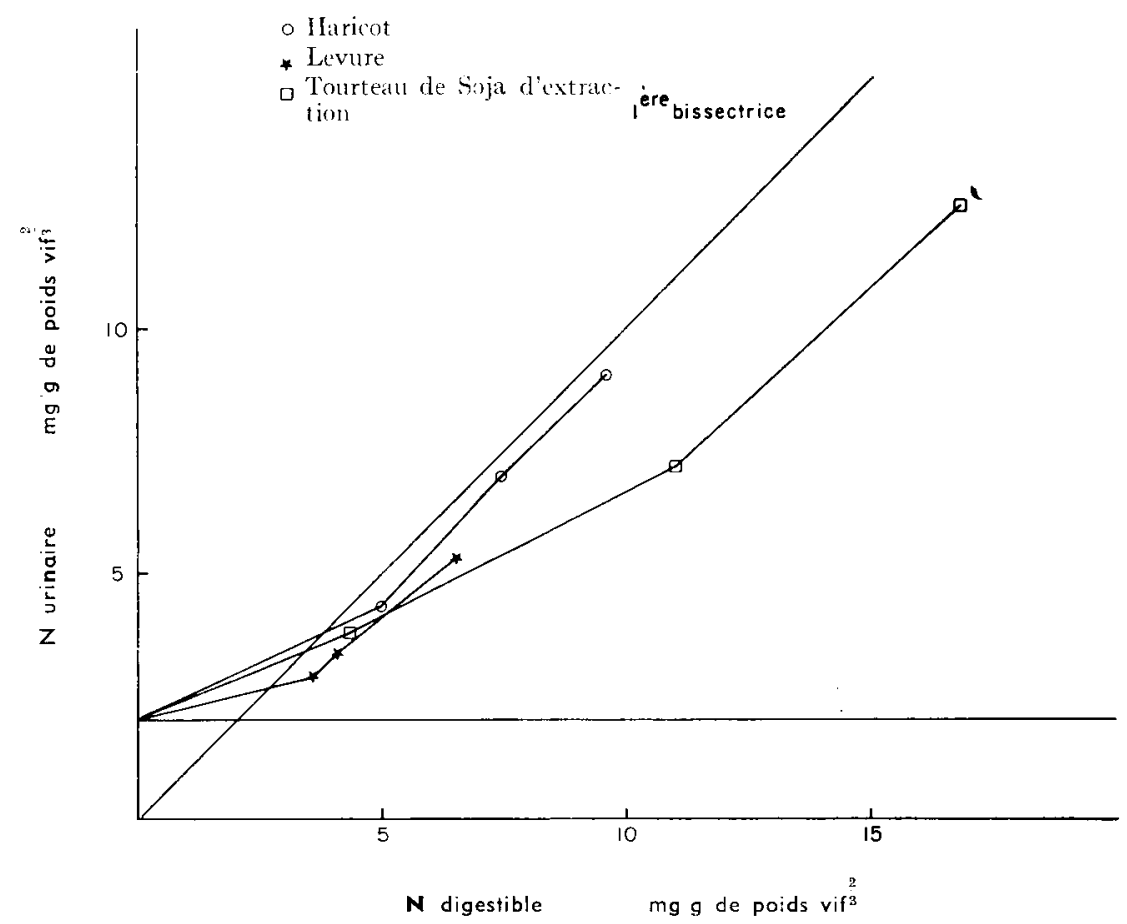

GRAPHugle I . - Détermination de lanabolisme protétqu jourialier maximal chez le Poulet pour diflérentes matières asotées; lorsque la courbe te l"excrétion devien parallite à la première bissectrice, on atteint lanabo. lisme maximal é tout l'azote suppiémentaire ingéré est excrélé.

D'apres HoIlls H. W., I955. Arch. Gefligelk. 19, $327 \cdot 353$.

le besoin quantitatif des M. A. T. pour une nature donnée de protéines, et de définir le besoin énergétique pour un besoin azoté précédemment déterminé.

Dans ce raisonnement, nous n'avons cependant pas tenu compte de la nature des éléments apportant les calories. Or nous avons vu que la mesure de la valeur des aliments en calories productives était souvent très délicate et n'avait de sens que si l'on précisait la composition du régime. Par la méthode classique, le rapport Cal/M. A. T., ne devrait donc être défini, que pour une protéine donnée et des aliments énergétiques définis.

Il ne faut pas cependant conclure à l'inutilité de la notion de rapport Cal/M. A. T. sous prétexte qu'on ne peut le définir parfaitement. Bien au contraire, il a permis une une amélioration considétable de l'efficacité des aliments, surtout dans la mesure où les matières premières constitutives sont parfaitement normalisées. On conçoit ainsi 
qu'aux Fitats-Unis où l'emploi d'un régime type simplifié à base de maïs et de soja est généralisé, l'application d'un même rapport Cal/M. A. T. est parfaitement valable.

Toutefois, pour retirer encore plus d'avantages de cette notion, on pourrait proposer une autre méthode de détermination. A notre avis, il conviendrait d'opérer en deux étapes. On pourrait en premier lieu définir le besoin azoté de l'animal par d'autres méthodes que la mesure de croissance, par exemple, comme l'a fait HoHLs (I955) qui mesure la rétention azotée de poulets recevant une protéine donnée à des taux variables.

Au fur et à mesure que le niveau azoté alimentaire s'élève, la rétention s'accroît jusqu'à une valeur qui ne peut être dépassée (graphique II). C'est à partir de cette valeur que HoHss définit " l'anabolisme protéique journalier maximal ". Chaque poussin possède donc une aptitude maximale à synthétiser des tissus protéiques qui est une constante de l'animal pour une protéine donnée. Il complète ses résultats en étudiant les facteurs de variation de cette constante protéique. L'anabolisme protéique maximal dépend de 1'âge de l'animal et de sa souche, d'une part et de la qualité de la protéine d'autre part.

Cette notion peut servir à une nouvelle définition du besoin azoté de l'animal qui serait la quantité minimale d'azote ingérée journellement qui permet d'atteindre la rétention maximale d'azote. Cette grandeur permet également de suivre la variation du besoin en fonction des différents facteurs liés à l'animal, d'une part, et au régime de l'autre. Malheureusement, HoHLs n'a, à notre connaissance, pas étudié directement la répercussion du taux énergétique sur cette valeur.

En second lieu, une fois déterminé, l'anabolisme protéique journalier maximal pour une protéine donnée, on pourrait fournir au poussin cette quantité d'azote séparée des éléments énergétiques et, mesurer la consommation spontanée d'énergie. I a méthode de CAI.E'T et al. (IgOI), que nous avons précédemment décrite, permet en effet de déterminer la quantité d'énergie que consommera le poussin recevant la protéine donnée en quantité donnée. Le nombre de calories fournies par un aliment de composition spécifiée, peut servir à la définition du besoin énergétique, dans la mesure où le poussin sait couvrir ce besoin. Ceci n'a pas été démontré, mais nous avons des raisons de croire la méthode valable, car dans les expériences citées ci-dessus, elle a toujours fourni le développement pondéral le meilleur et la rétention azotée la plus forte (mesurée par la " Net Protein Utilization »).

\section{CONCLUSION}

Nous avons rapporté les principaux travaux effectués sur la relation existant entre la nutrition azotée et la nutrition énergétique du poulet. Én particulier, nous avons analysé les données concernant le rapport Ca1/M. A. T. optimal en essayant de dégager les facteurs de ses variations.

On peut considérer que ce rapport représente le quotient de deux besoins. En ce sens il est d'abord fonction de l'animal : c'est ainsi qu'on doit l'adapter à l'âge, à la souche et, sans doute au sexe du poussin, aussi bien qu'à l'état d'engraissement que l'on désire obtenir.

Ce rapport est malgré tout un moyen assez grossier d'exprimer ces besoins. Les interactions matières azotées - énergie existant au niveau de l'appétence que de la 
digestion ou du métabolisme font qu'il est impossible de définir un rapport Cal/M. A. 'T. optimal sans préciser à la fois le pourcentage et la nature des matières azotées et des matières énergétiques. Par contre, il nous semble possible de déterminer pour un poulet donné, à partir d'une protéine ou d'un mélange de protéines donnés : primo, un besoin azoté en prenant comme base l'anabolisme protéique journalier maximal, secondo, un rapport Cal/M. A. T. optimal correspondant à une quantité de M. A. T. égale à cet anabolisme et au nombre de calories, d'origine donnée, que choisit le poulet.

Dans un autre domaine, il nous est apparu que le rapport Cal/M. A. T. est un critère intéressant du point de vue zootechnique. On peut en effet, dans une certaine mesure économiser ces M. A. 'T. en dépensant davantage d'énergie, sans modifier la croissance des animaux de façon appréciable. On peut également arriver à un résultat semblable en employant des régimes hyperénergétiques puisqu'il faut, dans ce cas, élever le taux énergétique plus rapidement que le taux azoté.

Ces deux pratiques ont l'une et l'autre pour conséquence un engraissement accru des animaux. Autrement dit, on peut influencer assez largement la composition des carcasses par l'alimentation en énergie et protéines, ce qui est particulièrement important pour la finition du poulet de chair.

$$
\text { Reçu en novembre } 196 \mathrm{I} \text {. }
$$

\section{SUMMARY}

VARIATIONS IN THE CALORIE/PROTEIN RATIO IN CHICKEN FEEDS

\section{INTRODLCTION}

I. IMPORTANCE OF THE C/P RATIO.

$1^{0}$ Influence of $\mathrm{C} / \mathrm{P}$ ratio on growth rate;

$2^{\text {o }}$ Influence of $\mathrm{C} / \mathrm{P}$ ratio on the efficiency of the different feed constituents ;

a) protein efficiency ;

b) caloric efficiency ;

c) reciprocal sparing effect of calorie and protein;

d) significance of the feed efficiency.

II. OPTIMAL C/P VARIATIONS :

A. - Factors related to the bird:

$I^{\prime \prime}$ age ;

$2^{0}$ sexe :

$3^{0}$ genetic origin.

B. - - Factors related to the diet

Quantitative aspect

$1^{\circ}$ Influence of the absolute value of the terms of the ratio on its optimal value for :

a) growth ;

b) feed efficiency ;

c) body composition.

$2^{\circ}$ Specitic action of the energy level

a) action on growth:

b) interpretation ;

c) conclusion.

Qualitative aspect :

$1^{0}$ Rôle of the nature of the protein ; 
$2^{0}-$ Rôle of the nature of energy products.

a) position of the probleme;

b) unreliability of the method for measuring productive energy of fats. aa) Digestibility of fats, " sensu stricto";

influence of fat level;

influence of the nature of fats ;

influence of melting point ;

influence of degree of esterification.

bb) Role of non-fatty constituents of the feed on fat digestibility;

c) specific action of fats;

d) differences in the metabolic role of carbohydrates and lipids.

Conclusion.

$3^{\circ}$ Discussion ;

\section{RÉFÉRENCES BIBLIOGRAPHIQUES}

Abraham J., Calet C., Rerat A., Jacquot R., 196r. Solidarité des besoins énergétique et protéique de croissance : l'ajustement sjontané des calories et des protides C. R. Acad. Sci., 253, 2768-2770.

ALmousst H. J., 1957. Proteins and amino acids in animal nutrition. U. S. Indust. chem. Co. pp. I9.

BALDini J. T., RosEnberg H. R., 1955. The effect of productive energy level of the diet on the methionine requirement of the chick. Poult. Sci., 34, I301-1 307.

Baldini J. T., Rosenberg H. R., r957. The effect of caloric source in chick diet on growth, feed utilization and body composition. Poult. Sci., 36, 432-435.

Barnes R. H., Bosshardt D. K., 1946. The evaluation of protein quality in normal animal. Ann. N. Y. Ac. Sci., 47, 279 .

Barnes R. H., Tuthill S., Kwong E., Fiala G., I959. Effects of the prevention of coprophagy in the rat. V. lissential fatty acid deficiency. J. Nutr., 68, I 2 I-I 30 .

Biel.y J., Marci B., I954. Fat studies in poultry. 2. Fat supplements in chick and poult rations. Poult Sci., 33, I 220-1 227 .

Brely J., Marcir B., I957. Fat studies in poultry. 7. Fat and nitrogen retention in chicks fed diets containing different levels of fat and protein. Poull. Sci., 36, I235-г240.

Brette A., Pexchinat A., Wottling: H., ig60. Supplémentation en amino acides des aliments pour ponlets. - Techniques et travaux expérimentaux. Cahier $\mathrm{n}^{0} 4$, AEC. Commentry (Allier), 22 I-229.

Bruegghann J., Barti K., I959. Ixperimentelle Studien zur Verwendung von Schlachtfetten in Geflïgelrationen. Arch. Gelligelk., 23, i 2-21.

Calet C., Françols A., JacQuot R., i957. Influence comparée de lauréomycine, du sulfate de cuivre, du tanin et de la lécithine sur la croissance pondérale de rat blanc et sa composition corporelle. Arch Sci. Physiol., 11, 21 3-222.

Calet C., Jocaxinet C., Barator J., I96I. Variation de la consommation spontanée d'énergie du poussin en fonction de la nature des matières azotées du rérime. Inn.. Biol. anim. Bioch. Biophys., 1, 5-9.

Carrol. K. K., Richariss J. F., 1958. Factors affecting digestibility of fatty acids in the rat. J. Nutr., 64, $41 \mathrm{I}-724$.

CARroli. K. K., I959. The growth-retarding effect of erucir, acid and its relation to requirements for fatsoluble vitamins. Canad. J. Biochen. Physiol., 37, 731-740.

Conbs (i. F., 1957. Proc. Univ. Maryland Nutr. Conf. Ficed Manufac. 55-65, cité par Calet C., Baratou J., 1960. Techniques et travaux expérimentaux. Cahier n10 4, AEC, Commentry (Allier), 26r-67.

Combs G. F., Quillin F. C., Helbacka N. V., Caskey C. D., I958. Studies on high efficiency broiler rations. Feedstuffs, 30, (28), i 8-22.

Conbs G. F., I 959 . Maryland Conference focuses attention on recent findings in poultry, animal feeding, Feedstufls, 31, (12), $52-56$.

Dansky I. M., IIrte F. W., I95г. The effect of energy level and physical nature of the diet on growth and body composition of chicks. Poult. Sci., 30, gro.

Davis II. J., Upp C. W., I941. Studies on the fat requirements of the domestic fowl. Poult. Sci., 20. 459 .

Donaldson W. E., Combs G. F., Romoser G. L., Supplee W. C., 1955. - Body composition, energy intake, feed efficiency, growth rate, and feather condition of growing chickens as influenced by calorieprotein ratio of the ration. Poult. Sci., 34, i 190 .

Donaldson W. E., Combs G. F., Romoser G. L., 1956. Studies on energy levels in poultry rations. I. The effect of calorie-protein ratio of the ration on growth, nutrient utilization and body composition. Poult. Sci., 35, r 100-1 ro5.

Donovan G. A., WARDen W. K., Reynolds W. M., I958. A comparison of unidentified growth factor sources for turkeys and influence of calorie-protein ratio on response. Poult. Sc2-i, 37, 42428 . 
Duckworth J., Naftalin J. M., Datgario A. C., I950. The digestibility of linseed oil and mutton fat by chicks. J. Agric. Sci., 40, 39-43.

Ferrando R., Brion A., I958. Problèmes soulevés par l'adjonction de graisses dans la ration des volailles. Journées d'études de l'École nat. v'étér. d'Aljort, 35-52.

Ferranijo R., Coleov J., ig6o. Problemes essentiels et aspects nouveaux de l'alimentation du ponlel de chair. Bull. tech. Ing. Serv. agric., 152, $49 \mathrm{I}-99$.

Forbes E. 13., Swift R. W., Elliott R. F., JAMkS W. II., ig46. Relation of fat to economy and food utilization. I et II. J. Nutr., 31, 203-212, 213-227.

FRAPS G. S., I943. Relation of the protein, fat, and energy of the ration to the composition of ohickens. Poult. Sci, 22, $42 \mathrm{I}-424$.

Fraps G. S., 1946. Composition and productive energy of poultry feed and ration. Texas Exper. Stal. Bull., $\mathrm{n}^{0} 678$.

Gordon R. S., Maddy K. II., Machlis L. J., I958. Proportion of dietary energy content to protein content as the prime determinant of the amino acid requirements of the young chicken. Ponlt. Sci., 37, I 208.

HEBFR'T B. A., BRunSON C. C., I957. 'The effects of diethylstilbestrol, testostérone, thiouracil, and thyroprotein on the chemical composition of broiler carcasses. Poult. Sci., 36, 898-904.

Hill F. W., DANSKY L. M., 1950. Studies on the protein requirements of chicks and its relation to dietary energy level. Poult. Sci., 29, 763 .

HInl F. W., Dansky L. M., 1954. Studies on the energy requirements of chickens. I. The effect of dietary energy level on growth and feed consumption. Ponlt. Sci, 33, i 2 - тा 9.

IILL F. W., Anderson D. J.., 1958. Comparison of metabolisable energy and productive energy determinations with the chicks. J. Nutr., 64, $587-603$.

Honls H. W., r955. Der maximal mögliche tïgliche Ëiweissansatz von Legnornkiken verschiedener Alters. Arch. Geflitgelk., 19, 327-353.

Houls H. W., 195\%. Der Einfluss des Kalorieneiweissverhältnisses auf die Rohverwertung be jwachsenden Hïhnern. Arch. Geflïgelk., 22, 395-415.

Honls II. W., I959. Mastfiihigkeitsvergleich zwischen Leghorn u. Cornish X White Rock-Kreuzung bei drei verschiedenen Futtermischungen. Arch. Gellïgelk., 23, 338-348.

Hohls H. W., 1960. Mastversuche mit Fettzugaben bei Leghorn und Nichols g/ros. Arch. Geflügelk., 24, $103-125$.

Kare II. R., Black R., Alissox E. G., I957. The sense of taste in the fowl. Poult. Sci., 36, r29-138.

Klopotowski T., Smietanska Z., Ig6o. The increased lipid synthesis in the livers of rats fed a diet containing soyabean oil. Acta Biochim. Polon., 7, $2 \mathrm{I}-28$.

LeONG K. C., Sunde M. I.., JiRD 11. R., Elvenjem C. A., I955. Effect of energy : protein ratio on growth rate, efficiency, feathering and fat deposition in chickens. Poult. Sci., 34, I 206-I 207.

IJEONG K. C., Sunde M. L., 13IRD II. R., JitueHJEM C. A., I959. Interrelationships among dietary energy, protein, and amino acids for chickens. Poult. Sci., 38, I $267-$ I284.

Leroy A. M., I954. Etilisation de l'énergie des aliments par les animaux. Ann. Zoolech., 3, 337-372.

Levelle G. A., Fisher H., I958. The amino acid requirements for maintenance in the adult rooster. J. Nutr., 66, 44I-453.

Melden W. J., Hill F. W., Dukes H. H., I954. Studies on the energy requirement of chickens. 2. Effect of dietary energy level on the basal metabolism of growing chickens. Poult. Sci., 33, 79 I-798.

Mraz F. R., Boucher R. V., MCCARTnky M. G., 1957. The influence of the energy : volume ratio on growth response in chickens. Poult. Sci., 36, 1217-1221.

Mra7, F. R., Boccher R. V., MCCARTAEY M. G., 1958. The influence of dietary energy and protein on growth response in chickens. Poult. Sci., 37, 1308-1313.

Pepper W. I., Slinger S. J., Snyder I.. S., I953. Increasing the energy content of broiler diets high in wheat. Poult. Sci., 32, 920-921.

Peterson D. W., Grau C. R., Peek N. F., 1952. Growth and food utilization with diets of varying protein and cellulose levels. Poull. Sci., 31, $93^{\text {I. }}$

Potter L. M., Matterson L. D., Carlson D., Singsen L. P., i956. Studies on the effect of varying protein levels and calorie : protein ratios in poultry rations on growth and feed efficiency. Poult. Sci., 35, II 65.

Raulin J., Clement J., Blum J. C., r959. Étude de la toxicité de l'association alimentaire acides gras libres et cholestérol. Mlodalités d'action de l'extra-choline et rôle de la sécrétion biliaire. Arch. Sci. Physiol., 13, $79-98$.

Reiser R., Covch J. R., 1949. The essential role of fat in the rations of growing chicks. Poult. Sci., $28,78 \mathrm{r}$.

Renner R., Hill F. W., I958. Metabolizable energy values of fats and fatty acids for chickens. Proc. Cornell Nutr. Conf. Lieed Manuf., 95-100.

Reyntens N., ig60. Influence des calories et des protéines dans l'alimentation des poulets. I confér. Europ. avic., I960. Utrecht. 
Ringrose R. C., Gordon R. S., Maddy K. H., Sanson W. R., Potter L. M., 1955. Factors affecting the response of broilers to methionine hydroxyanalogue. Poult. Sci., 34, I218.

Rose W. C., Coon M. J., Lambert G. F., I954. The amino acid requirements of man 6 . The role of the calorie intake. J. Biol. Chem., 210, 33.

Rosenberg H. R., Baldini J. T., I957. Effect of dietary protein level on the methionine-energy relationship in broiler diets. Poult. $S_{c i}, 36,247^{-2} 5^{2}$.

ScotT H. M., Matterson L. D., Singsen E. P., I947. Nutritional factors influencing growth and efficiency of feed utilization. I. The effect of the source of carbohydrate. Ponlt. Sci., 26, 554 .

ScotT H. M., Sims L. C., Staheli D. L., 1955. The effect of varying protein and energy on the performance of chicks. Poult. Sci., 34, I220.

Shutze J. V., Thornton P. A., Moreng R. E., I958. Protein energy relationships as affected by sex and management. Poult. Sci., 37, 1063-1070.

Siedler A. J., Schweigert B. S., I953. Effect of feeding graded levels of fat with and without choline and antibiotic $+\mathrm{B}_{12}$ supplements to chicks. Poult. Sci., 32, 449-454.

Sunoe M. L., I956 a. A relationship between protein level and energy level in chick rations. Poult. Sci., $35,350-354$.

Sunde M. L., I956 b. The effect of fat and fatty acids in chick rations. Poult. Sci., 35, 362*368.

Swanson E., r959. Protein energy an the metabolism of nitrogen. Protein and Aminoacid Nutrition, Academic Press, I95-224.

Thompson W. S. T., Munro H. Y., 1955. The relationship of carbohydrate metabolism to protein metabolims. J. Nutr., 56, I39-150.

Twining P. F., Romoser G. L., Combs G. F., I955. Studies on methionine requirements of growing chickens. Poult. Sci., 34, I225-26.

Vondell R. M., Ringrose R. C., 1958. The effect fo protein and fat levels and calorie to protein ratio upon performance of broilers. Poult. Sci., 37, I47-I5I.

Waibel P., I960. Protein and amino acid nutrition of turkeys. Feedstuffs, 23, (23), 46-52.

Williams M. A., GRAU C. R., 1953. Effects of variations in dietary energy on food intake and utilization of lysine deficient protein. Poult. Sci., 32, 928.

Williams W. P., Davies R. E., Couch J. R., r959. Digestibility coefficients of certain fats and oils in chick diets as influenced by the basal ration. Poult. Sci., 38, 1260.

Yacowitz H., 1953. Supplementation of corn-soybean oil meal with penicillin and various fats. Poult. Sci., 32, 930.

Yoshida A., Harper A. E., Elvehjem C. A., I957. Effects of protein per caloric ratio and dietary level of fat on calorie and protein utilization. $J$. Nulr., 63,555 . 